\title{
IL-26 contributes to host defense against intracellular bacteria
}

\author{
Angeline Tilly Dang, ${ }^{1,2}$ Rosane M.B. Teles, ${ }^{1}$ David I. Weiss, ${ }^{1,2}$ Kislay Parvatiyar, ${ }^{2}$ Euzenir N. Sarno, ${ }^{3}$ Maria T. Ochoa, ${ }^{4}$ \\ Genhong Cheng, ${ }^{2}$ Michel Gilliet, ${ }^{5}$ Barry R. Bloom, ${ }^{6}$ and Robert L. Modlin ${ }^{1,2}$ \\ 'Division of Dermatology, Department of Medicine, 2Department of Microbiology, Immunology and Molecular Genetics, David Geffen School of Medicine at UCLA, Los Angeles, California, USA. \\ ${ }^{3}$ Leprosy Laboratory, Oswaldo Cruz Foundation, Rio de Janeiro, Brazil. ${ }^{4}$ Department of Dermatology, University of Southern California School of Medicine, Los Angeles, California, USA. \\ ${ }^{5}$ Department of Medicine, Dermatology Service, Lausanne University Hospital of Lausanne, Lausanne, Switzerland. ${ }^{6}$ Harvard School of Public Health, Boston, Massachusetts, USA.
}

\begin{abstract}
IL-26 is an antimicrobial protein secreted by Th17 cells that has the ability to directly kill extracellular bacteria. To ascertain whether IL-26 contributes to host defense against intracellular bacteria, we studied leprosy, caused by the obligate intracellular pathogen Mycobacterium leprae, as a model. Analysis of leprosy skin lesions by gene expression profiling and immunohistology revealed that IL-26 was more strongly expressed in lesions from the self-limited tuberculoid compared with expression in progressive lepromatous patients. IL-26 directly bound to $M$. leprae in axenic culture and reduced bacteria viability. Furthermore, IL-26, when added to human monocyte-derived macrophages infected with $M$. leprae, entered the infected cell, colocalized with the bacterium, and reduced bacteria viability. In addition, IL-26 induced autophagy via the cytoplasmic DNA receptor stimulator of IFN genes (STING), as well as fusion of phagosomes containing bacilli with lysosomal compartments. Altogether, our data suggest that the Th17 cytokine IL-26 contributes to host defense against intracellular bacteria.
\end{abstract}

\section{Introduction}

The gene encoding IL-26 was discovered in Herpesvirus saimiritransformed human $\mathrm{T}$ cells, with approximately $25 \%$ sequence homology and approximately $47 \%$ amino acid similarity to IL-10 (1). As such, IL-26 is considered a member of the IL-10 cytokine family, which includes IL-10, IL-19, IL-20, IL-22, and IL-24 (2, 3). The IL26 gene is located on chromosome 12q15, between IFNG and IL22. IL26 is highly conserved in mammalian species and more weakly similar to nonmammalian species. However, IL26 is absent in rodents, despite the presence of both IL-26 receptor genes (4-6). IL-26 contains 171 amino acids, including lysine or arginine 30 residues, forming 6 highly cationic $\alpha$-helices (1). Th17 cells are the main producers of IL-26 $(7,8)$, but some Th1 cells (9) and NK cells (10) are reported to produce this cytokine. Although monocytes (11) and macrophages (12) have been reported to release IL-26, the purity of the cell populations was not clearly stated.

The structure of IL-26, including the $\alpha$-helices, the amphipathic stretches, the clustering of cationic charges, and the formation of multimers, are hallmarks of naturally occurring antimicrobial peptides $(13,14)$. This led to the discovery that IL-26 is the only known Th17 cytokine with antimicrobial properties, with the ability to kill extracellular bacteria including Pseudomonas aeruginosa, Escherichia coli, Klebsiella pneumoniae, and Staphylococcus aureus

Authorship note: ATD, RMBT, and DW contributed equally to this work Conflict of interest: The authors have declared that no conflict of interest exists. Copyright: (C) 2019, The American Society for Clinical Investigation.

Submitted: December 28, 2017; Accepted: February 19, 2019; Published: April 2, 2019. Reference information: / Clin Invest. 2019;129(5):1926-1939.

https://doi.org/10.1172/JCI99550. that largely reside and replicate outside of cells $(3,9)$. Similar to other antimicrobial proteins, IL-26 reduced bacterial viability via direct binding to the bacterial cell wall, leading to pore formation and membrane disruption (9).

In addition to its direct antimicrobial activity, IL-26 was shown to signal through the IL-10R2/IL-20R1 heterodimeric receptor, with both subunits simultaneously expressed exclusively and constitutively by epithelial cells $(15,16)$. Despite sharing homology with the antiinflammatory cytokine IL-10 and a receptor subunit with the IL-10 receptor, the majority of evidence indicates that IL-26 not only initiates inflammation but also propels it (17-19). IL-26 was found to induce the expression of IL-10, TNF- $\alpha$, and IL- 8 in epithelial cells, as well as inhibit their proliferation (20). IL-26 can also drive inflammation by acting as a potent chemoattractant for neutrophils (12), which subsequently can be activated to release inflammatory cytokines, proteases, and antimicrobial peptides (21). One report indicated that, although IL-26 by itself induced cytokine responses in bronchoalveolar lavage (BAL) cells, it inhibited the release of myeloperoxidase from BAL cells and neutrophils by less than $25 \%$ (12).

In the present study, we investigated whether IL-26 contributes to host defense against the obligate intracellular bacterium by studying human leprosy as a model. The disease, caused by the Mycobacterium leprae, forms a spectrum, in which the clinical presentation correlates with the effectiveness of the immune response. In the self-limited tuberculoid leprosy (T-lep) form, the bacteria are eliminated, whereas, in the disseminated lepromatous (L-lep) form, bacilli-laden macrophages are prominent, indicating an inability to contain the infection. The results provide evidence that IL-26 may act intracellularly as well as extracellularly to kill microbial pathogens. 

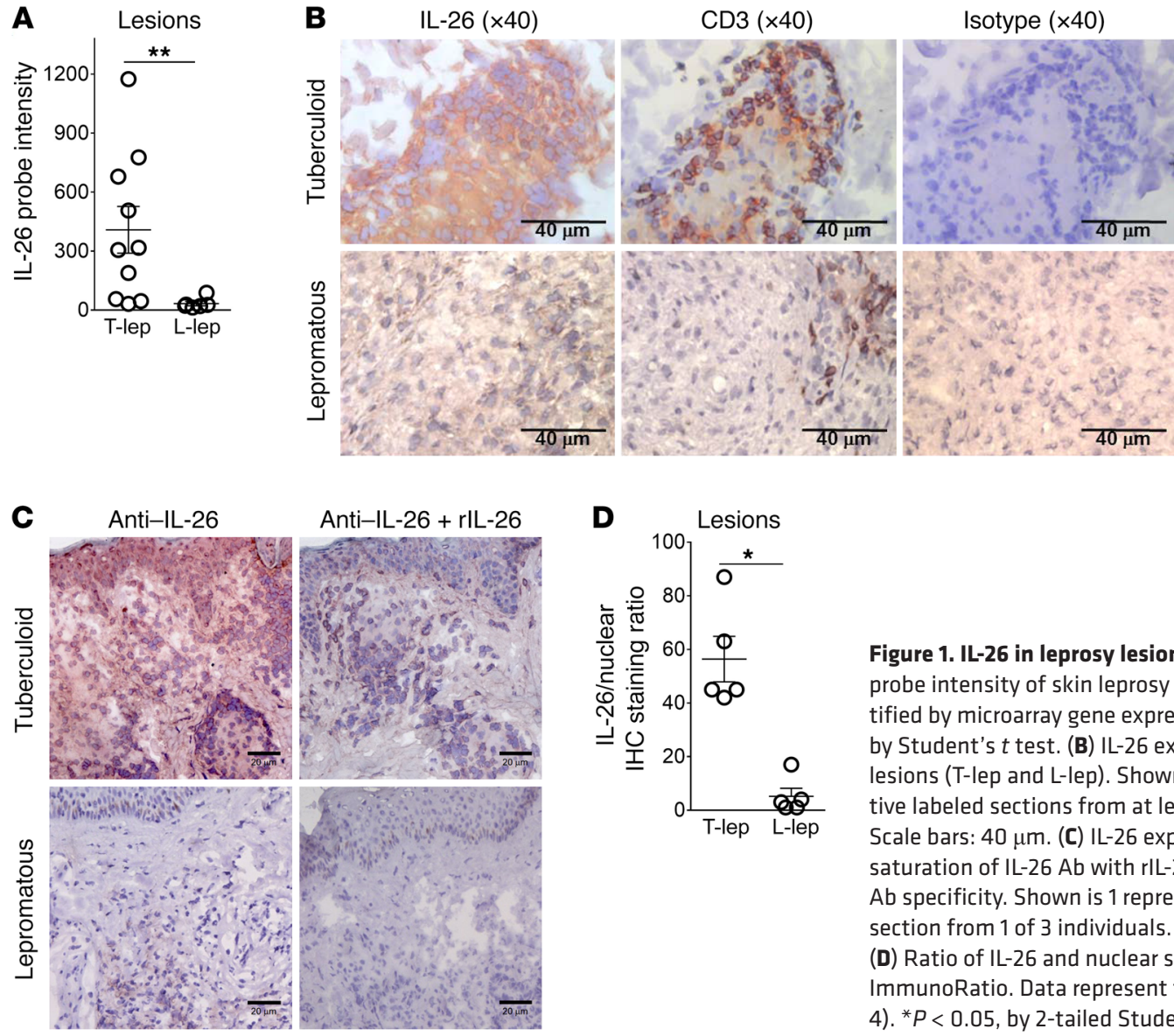

\begin{abstract}
Figure 1. IL-26 in leprosy lesions. (A) IL26 mRNA probe intensity of skin leprosy lesions was quantified by microarray gene expression. ${ }^{* *} P<0.01$, by Student's $t$ test. (B) IL-26 expression in leprosy lesions (T-lep and L-lep). Shown are 2 representative labeled sections from at least 4 individuals. Scale bars: $40 \mu \mathrm{m}$. (C) IL-26 expression following saturation of IL-26 Ab with rIL-26, demonstrating Ab specificity. Shown is 1 representative labeled section from 1 of 3 individuals. Scale bars: $20 \mu \mathrm{m}$. (D) Ratio of IL-26 and nuclear staining quantified by ImmunoRatio. Data represent the mean \pm SEM ( $n=$ 4). ${ }^{*} P<0.05$, by 2 -tailed Student's $t$ test.
\end{abstract}

\title{
Results
}

IL-26 expression in human leprosy. We mined the gene expression data derived from leprosy lesions (22) to determine whether there was differential expression of IL26 mRNA across the spectrum of disease. This analysis revealed that IL26 mRNA levels were significantly greater in the skin biopsy specimens from T-lep patients compared with those from L-lep patients (Figure 1A), with probe intensities of $408 \pm 118$ in the gene expression data from T-lep lesions versus $33 \pm 11$ from L-lep lesions $(P<0.05)$. In 7 of $10 \mathrm{spec}-$ imens from T-lep donors, the expression of IL26 mRNA was greater than in the specimens from all the L-lep donors.

We further assessed the differences in IL-26 expression in leprosy lesions by immunohistochemical analysis with an anti-IL-26 monoclonal Ab. IL-26 protein expression was abundant in T-lep lesions, throughout the granuloma, and particularly strong in the vicinity of small lymphoid cells, but was also diffusely present near larger mononuclear myeloid cells (Figure 1B). In contrast, IL-26 expression was sparse throughout the L-lep lesions. We also assessed the specificity of the anti-IL-26 $\mathrm{mAb}$ by adding recombinant monomers of IL-26 protein to the Ab prior to tissue staining. Saturation of the mAb by recombinant IL-26 reduced the signal intensity in T-lep lesions, demonstrating specificity of the Ab to the protein (Figure 1C). Anti-CD3 served as a positive control. All isotype controls were consistently negative. Quantification was performed using the online software ImmunoRatio (23) to measure the IL-26 to nuclear staining ratio, which provides a measure of IL-26 expression. This ratio is not intended as a measure of IL-26-

expressing cells, as we could not determine whether IL-26 was being produced or taken up by individual cells. The IL-26 to nuclear staining ratio was approximately 10-fold higher in T-lep lesions than in L-lep lesions: $56 \pm 8.5$ vs. $5.2 \pm 3.0(P<0.05$, Figure $1 D)$.

We used confocal laser microscopy to determine the relative localization of IL-26 in relation to T cells and macrophages within leprosy lesions. Again, we observed that IL-26 expression was greater in T-lep lesions than in L-lep lesions, colocalizing more frequently with $\mathrm{CD}^{+} \mathrm{T}$ cells in T-lep versus L-lep lesions (Figure 2, A and B) and, to a lesser extent, with $\mathrm{CD} 8^{+} \mathrm{T}$ cells (Figure $2, \mathrm{C}$ and $\mathrm{D}$ ). The frequency of $\mathrm{CD}^{+} \mathrm{T}$ cells that colocalized with IL-26 was greater in T-lep lesions, at $26.7 \% \pm 3.7 \%$ compared with $3.2 \% \pm 0.7 \%$ in L-lep lesions $\left(P<0.01\right.$, Figure 2B). In addition, the frequency of $\mathrm{CD}^{+}$ $\mathrm{T}$ cells that colocalized with IL-26 was greater in T-lep lesions, at $17.5 \% \pm 1.2 \%$, compared with L-lep lesions, which had $7.2 \% \pm 1.3 \%$ colocalization $(P<0.05$, Figure 2D). In a simultaneous comparison of the levels of colocalization of IL- 26 with the $\mathrm{CD} 4^{+}$and $\mathrm{CD} 8^{+} \mathrm{T}$ cell markers, we found that IL-26 colocalized significantly more with $\mathrm{CD}^{+}$than with $\mathrm{CD}^{+} \mathrm{T}$ cells $(P<0.05$, Figure $2 \mathrm{E})$. Additionally, we analyzed the relative location of IL-26 in relation to $\mathrm{CD} 68^{+}$macrophages in leprosy lesions and found that IL-26 was coexpressed with CD68 in both types of lesions (Figure 3A). However, the degree of colocalization between IL-26 and the CD68 marker was also higher in T-lep lesions than in L-lep lesions $(16.8 \% \pm 2.1 \%$ versus $8.3 \%$ $\pm 1.1 \%$, respectively) $(P<0.05$, Figure $3 \mathrm{~B})$. We also observed areas in T-lep granulomas in which IL-26 colocalized with both $\mathrm{CD}^{+}$ and $\mathrm{CD}^{+} 8^{+}$cells (Figure $3 \mathrm{C}$ ). In a simultaneous comparison of the 
A

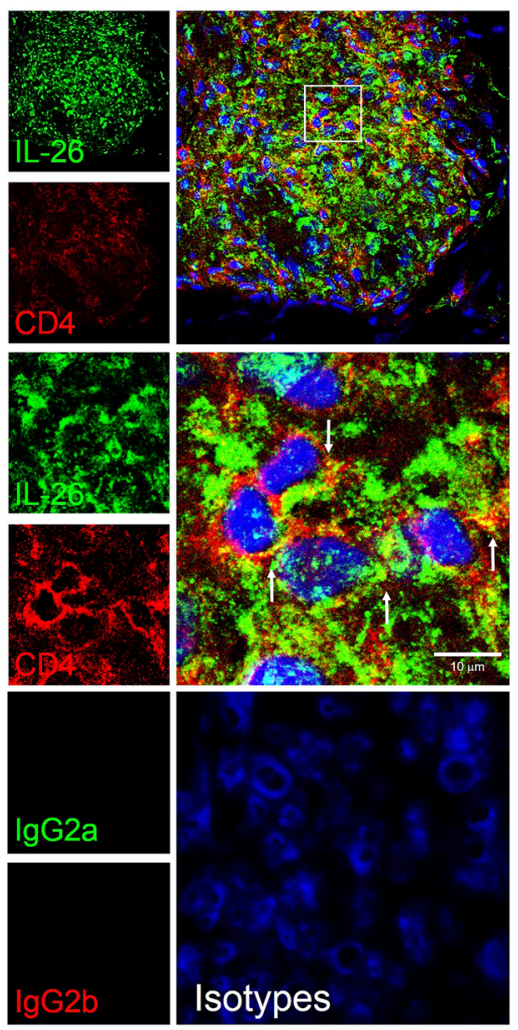

C

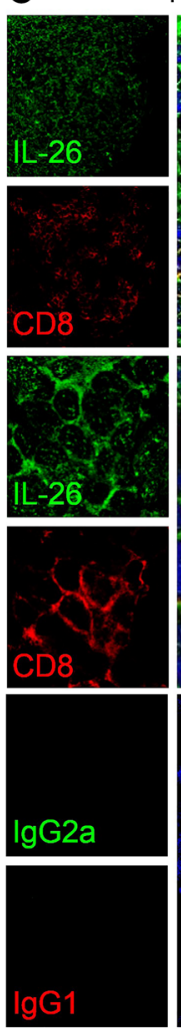

Tuberculoid

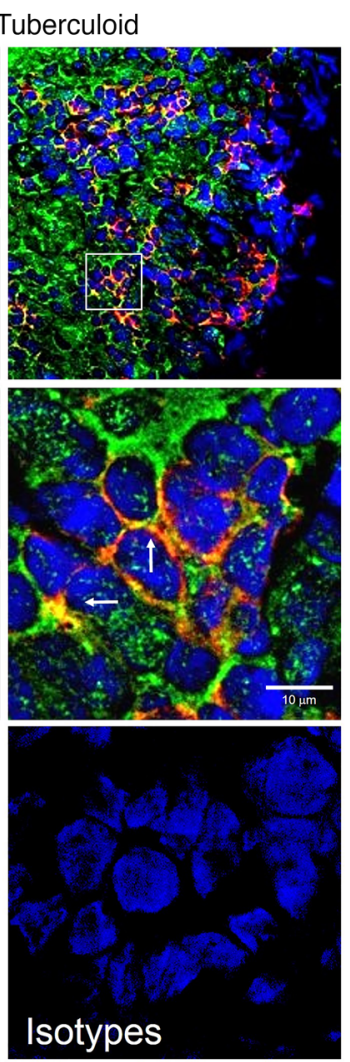

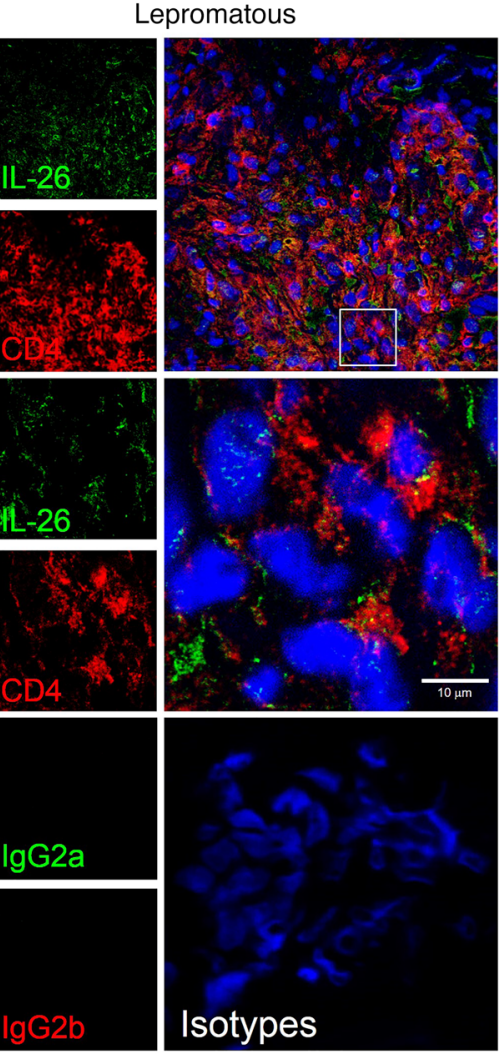

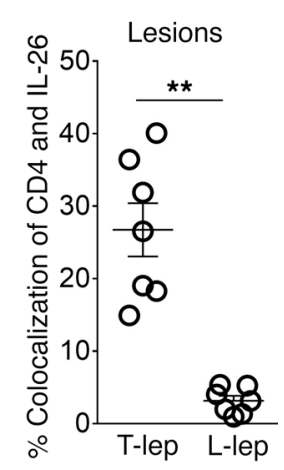

Figure 2. IL-26 colocalization with CD4 and CD8. (A) Immunofluorescence of IL-26 (green), CD4 (red), and nuclei (DAPI, blue) in T-lep and L-lep lesions. Data are representative of 4 individual samples. Original magnification, $\times 20$. Scale bars: $10 \mu \mathrm{m}$ (enlarged insets). (B) Colocalization of CD4 and IL-26, as determined by Image). Data represent the mean $\pm \operatorname{SEM}(n=4) .{ }^{* *} P<0.01$, by 2-tailed Student's $t$ test. (C) Immunofluorescence of IL-26 (green), CD8 (red), and nuclei (DAPI, blue) in T-lep and L-lep lesions. White arrows indicate colocalization of green and red (yielding yellow). Original magnification, $\times 20$. Scale bars: $10 \mu \mathrm{m}$ (enlarged insets). (D) Colocalization of CD8 and IL-26, as determined by ImageJ. Data represent the mean $\pm \operatorname{SEM}(n=4) .{ }^{*} P<$ 0.05 , by 2 -tailed Student's $t$ test. (E) Comparison of CD4 or CD8 colocalization with IL-26 in T-lep using Image). Data represent the mean $\pm \operatorname{SEM}(n=4)$. ${ }^{*} P<0.05$, by 2-tailed Student's $t$ test.

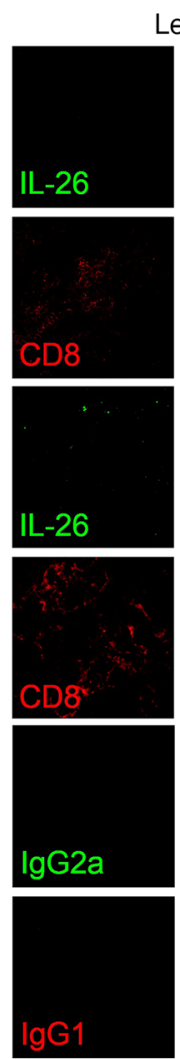

Lepromatous

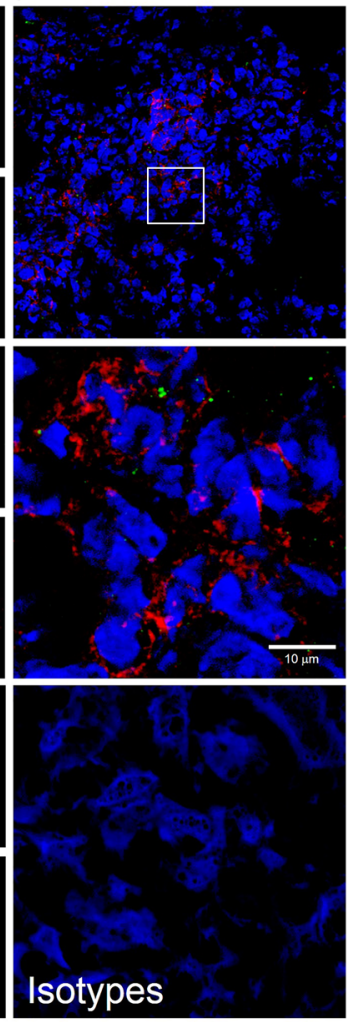

D

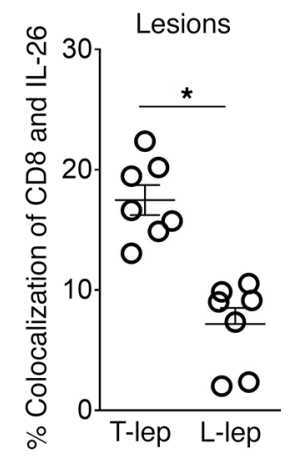

E Tuberculoid

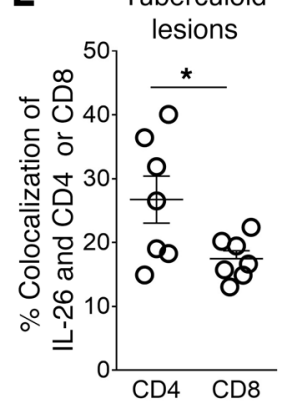

levels of colocalization of IL-26 with the CD4 and CD68 markers, we found that IL-26 colocalized significantly more with $\mathrm{CD} 4^{+}$than with $\mathrm{CD} 8^{+}(P<0.05$, Figure 3D). These data show the proximity of IL-26 to $\mathrm{CD}^{+}{ }^{+}$and $\mathrm{CD} 68^{+}$cells, but again, these data define the microanatomic location of IL-26 but cannot distinguish between production versus uptake of the protein.

IL-26 directly reduces the viability of mycobacteria in axenic conditions. As a first step toward defining the role of IL-26 against 
intracellular M. leprae, we determined whether IL-26 bound to the bacterium. M. leprae was incubated with recombinant IL-26 protein in axenic culture. After 6 hours, IL-26 was detected on the surface of some M. leprae bacilli (Figure 4, A and C). Quantification of confocal images confirmed that the majority of bacilli were bound by IL-26, $89.5 \% \pm 0.5 \%$, leaving $9.5 \% \pm 0.51 .1 \%$ free bacilli (Figure 4B). Analysis of confocal images using Imaris software revealed that the bacilli that were bound to IL-26 were larger in diameter, as measured by transverse width, compared with media controls. The width of the bacilli increased from $0.359 \pm 0.012 \mu \mathrm{m}$ in media-treated bacteria to $0.528 \pm 0.023 \mu \mathrm{m}$ in $1 \mu \mathrm{M}$ or $0.591 \pm$ $0.038 \mu \mathrm{m}$ in $2 \mu \mathrm{M}$ of IL-26-treated bacteria $(P<0.01$, Figure $4 \mathrm{D})$. The increased diameter is consistent with osmotic lysis of the bacteria, one mechanism by which antimicrobial peptides mediate an antimicrobial response.

Although $M$. leprae does not grow in culture, it is possible to measure bacterial viability by quantitative PCR (qPCR) using the ratio of $M$. leprae $16 \mathrm{~S}$ rRNA to the repetitive element DNA RLEP as a proxy for transcriptional activity, as previously reported (22, $24,25)$. We studied the effects of IL-26 in axenic culture to determine whether it further reduced bacterial viability and found that IL-26 treatment of $M$. leprae for 3 days reduced the viability of the bacteria in a dose-dependent manner (Figure 5A). The observed antimicrobial activity was diminished when IL-26 was denatured prior to addition to $M$. leprae (Figure 5B). Similarly, IL-26 inhibited the growth of Mycobacterium tuberculosis H37Ra in axenic culture, quantified by a CFU assay (Figure $5 \mathrm{C}$ ). These findings reveal that IL-26 binds directly to mycobacteria and mediates a reduction in bacteria viability.

IL-26 is taken up by monocyte-derived macrophages and colocalizes with $M$. leprae. During $M$. leprae infection, bacilli can be found in a variety of tissues and cell types but are predominantly located in macrophages $(26,27)$. Although it has been reported that IL-26 concentrations up to $10 \mu \mathrm{M}$ did not affect the viability of human primary human immune cells (9), we specifically assessed the cytotoxic effects of IL-26 on human monocyte-derived macrophages (MDMs). When we treated MDMs with increasing concentrations of IL-26, we observed some detachment of MDMs from the culture plates with the $2-\mu \mathrm{M}$ IL-26 treatment. With $5-\mu \mathrm{M}$ IL-26 treatment, the majority of cells were detached, and the cells that remained showed condensed nuclei and positive staining by TUNEL assay, indicative of cellular apoptosis (Figure 6A). Therefore, we stimulated MDMs with IL-26 at $2 \mu \mathrm{M}$ or less in all remaining experiments to ensure optimal cell viability.

Because $M$. leprae is an obligate intracellular organism, IL-26 must gain access to the intracellular compartments in which the bacteria reside in order to exert a direct antimicrobial activity. We therefore evaluated whether IL-26 entered MDMs and could be detected in the endosomal pathway in which $M$. leprae resides. Overnight treatment of MDMs with Alexa 488-labeled IL-26 resulted in the uptake of IL-26 and colocalization with lysosomal-associated membrane protein 1-positive (LAMP1-positive) compartments (Figure 6B). The colocalization of Alexa 488labeled IL-26 with LAMP1 was significantly greater than was seen with the addition of Alexa 488 dye alone, increasing from $2.3 \% \pm$ $0.4 \%$ colocalization in dye media alone to $19.6 \% \pm 3.3 \%$ in $1 \mu \mathrm{M}$ and $23 \% \pm 2.3 \%$ in $2 \mu \mathrm{M} \mathrm{IL}-26(P<0.01$, Figure $6 \mathrm{C})$. Furthermore, treatment of $M$. leprae-infected MDMs with Alexa 488-labeled IL-26 revealed colocalization of intracellular bacilli with IL-26 (Figure 6D). The degree of IL-26 colocalization with $M$. leprae was similar for treatment with the $1-\mu \mathrm{M}$ and $2-\mu \mathrm{M}$ concentrations of the cytokine: $11.9 \% \pm 1.5 \%$ and $11 \% \pm 0.6 \%$, respectively (Figure $6 \mathrm{E})$. Finally, we assessed whether pretreatment of MDMs with IL-26 affects the levels of infectivity by $M$. leprae. Although pretreatment of MDMs with IL-26 slightly reduced the infection efficiency of $M$. leprae, the differences in the percentages of $M$. lepraeinfected MDMs were not statistically significant (Figure 6F).

IL-26 induces autophagy and enhances bacterial trafficking to the lysosomes. The process of autophagy is required to overcome the ability of mycobacteria to block phagolysosomal fusion in infected macrophages in order to promote an effective antimicrobial response (28-30). Immunofluorescence data indicated a significant increase in the number of autophagosomes in MDMs following treatment with IL-26 compared with media control, as determined by quantification of LC3 puncta (Figure 7A). In media alone, we observed $8.3 \pm 1.3$ puncta per cell compared with IL-26 treatment, in which $57.9 \pm 5.5$ puncta per cell were detected $(P<$ 0.01 , Figure $7 \mathrm{~B}$ ). The ability of IL-26 to induce autophagy was confirmed by LC3 I to LC3 II conversion by immunoblotting (Figure 7C). Given that IL-26 has been shown to bind to DNA from dying cells in in vitro cultures and traffic this DNA to activate innate cytoplasmic receptors, e.g., stimulator of IFN genes (STING) $(9,31)$, we investigated whether IL-26-induced autophagy was STING dependent. We found that IL-26 induced autophagy in WT THP-1 cells but was strikingly diminished in THP-1 cells in which STING was ablated by stable KO. In contrast, rapamycin-induced autophagy was STING independent (Figure 7D). Overall, IL-26induced autophagy in THP-1 cells, as measured by the number of LC3 puncta per cell, was blocked by approximately $60 \%$ by the deletion of STING (Figure 7E). In contrast, only $25 \%$ of rapamycin-induced autophagy was STING dependent.

Having demonstrated that IL-26 induces autophagy and colocalizes with $M$. leprae bacilli during infection, we hypothesized that bacteria would localize to autophagosomes following IL-26 stimulation. We observed a significant increase in colocalization of $M$. leprae with the autophagosome marker LC3 and the lysosome marker LAMP1 (Figure 7F). We observed 5.1\% \pm 0.7\% colocalization between $M$. leprae and LC3 in the media control-treated MDMs versus $18.9 \% \pm 1.4 \%$ and $26 \% \pm 1.9 \%$ colocalization in the $1-\mu \mathrm{M}$ and $2-\mu \mathrm{M}$ IL-26-treated MDMs, respectively $(P<0.05$, Figure 7G). We noted a similar increase in the colocalization of M. leprae and LAMP1 following IL-26 stimulation, with $1.3 \% \pm$ $0.2 \%$ colocalization between $M$. leprae and LAMP1 in the media control-treated MDMs versus 3.3\% $\pm 0.4 \%$ and $6.4 \% \pm 0.8 \%$ colocalization in the $1-\mu \mathrm{M}$ and $2-\mu \mathrm{M}$ IL-26-treated MDMs, respectively $(P<0.01$, Figure $7 \mathrm{H})$. Although treatment with the higher concentration of IL-26 induced more $M$. leprae colocalization with LAMP1, we observed that IL-26 colocalization with LAMP1 remained comparable following treatment with either $1 \mu \mathrm{M}$ or 2 $\mu \mathrm{M}$ IL-26 (Figure 7, I and J), consistent with our observations with uninfected MDMs (Figure 6C). This suggests that the mechanism driving the increased traffic of $M$. leprae to LAMP1 compartments in infected MDMs is not through increased expression of LAMP1 but potentially through IL-26-induced autophagy. 
A
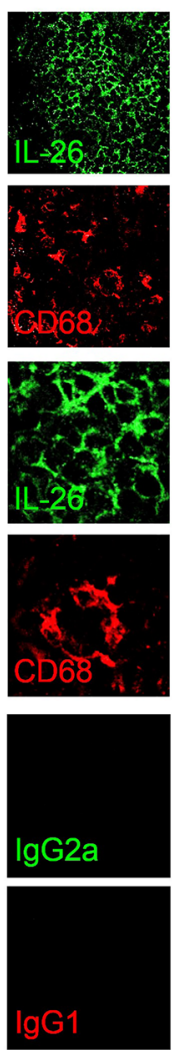

C
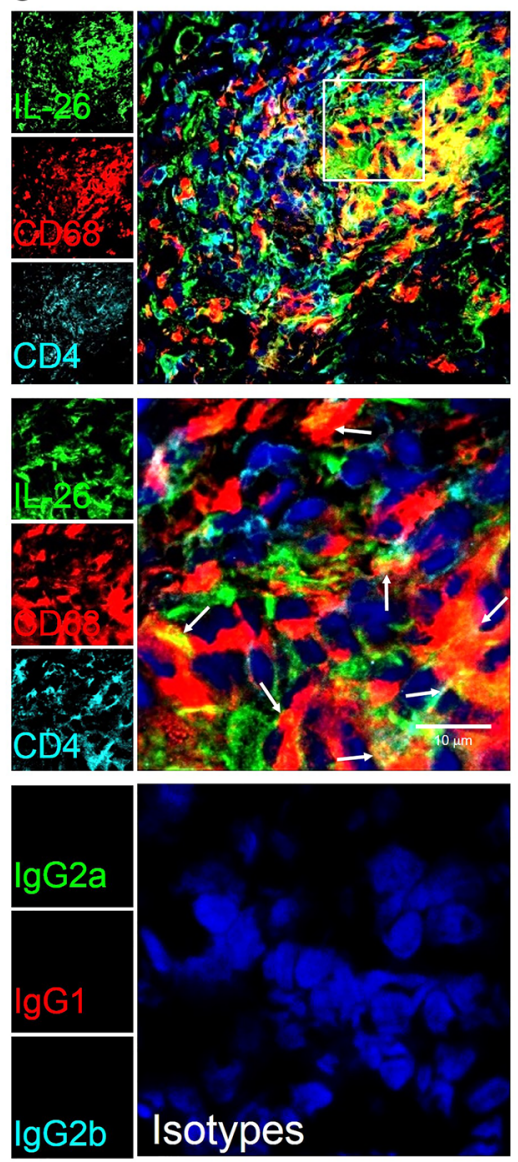

Tuberculoid
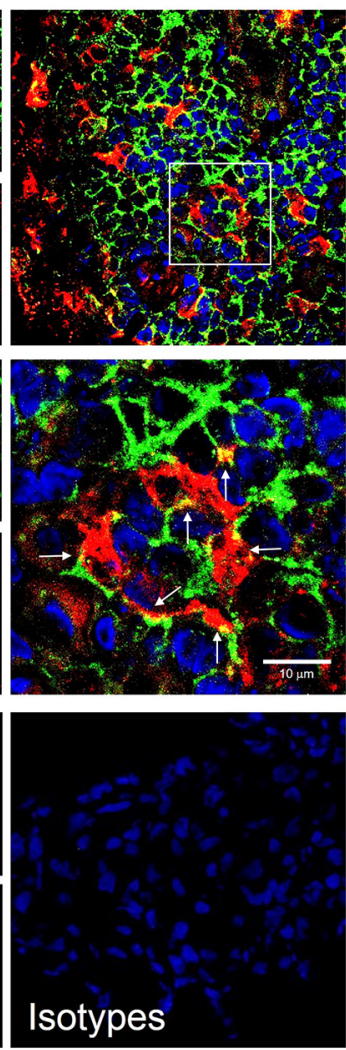

Lepromatous
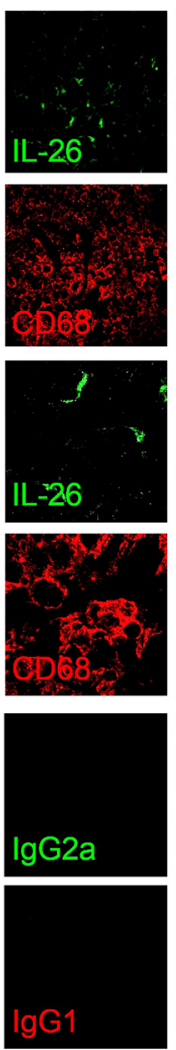

Isotypes
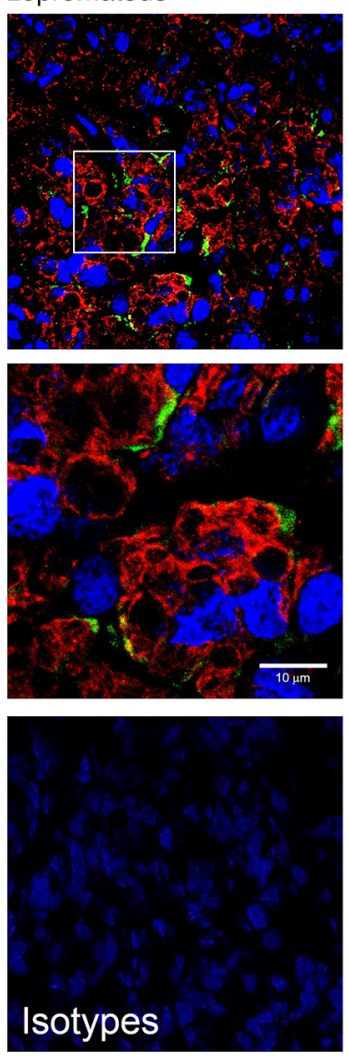

B

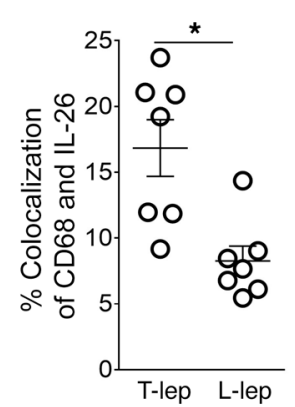

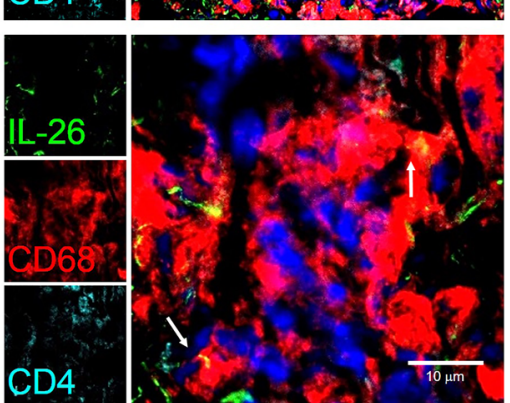

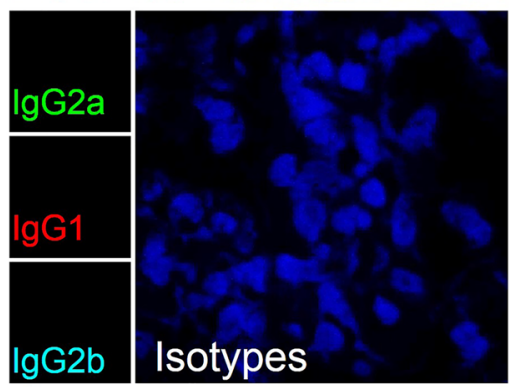

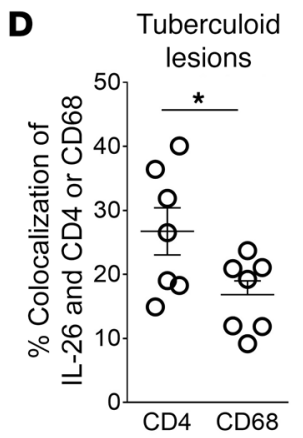

Figure 3. IL-26 colocalization with $\mathrm{CD}^{+} \mathbf{8}^{+}$macrophages in leprosy lesions. (A) Immunofluorescence of IL-26 (green), CD68 (red), and nuclei (DAPI, blue) in T-lep and L-lep lesions. White arrows indicate colocalization of IL-26 and CD68. Data are from 5 individual samples. Original magnification, $\times 20$. Scale bars: $10 \mu \mathrm{m}$ (enlarged insets). (B) Colocalization of IL-26 and CD68 in T-lep and L-lep lesions using Image). Data represent the mean $\pm \operatorname{SEM}(n=5) .{ }^{*} P$ $<0.05$, by 2 -tailed Student's $t$ test. (C) Immunofluorescence of IL-26 (green), CD68 (red), CD4 (cyan), and nuclei (DAPI, blue) in T-lep and L-lep lesions. Data are from 3 individual samples. White arrows indicate colocalization of IL-26 (green) and CD68 (red) (yielding yellow). Original magnification, $\times 20$. Scale bars: $10 \mu \mathrm{m}$ (enlarged insets). (D) Comparison of CD4 or CD68 colocalization with IL-26 in T-lep using ImageJ. Data represent the mean \pm SEM $(n=4)$. ${ }^{*} P<0.05$, by 2 -tailed Student's $t$ test. 
A

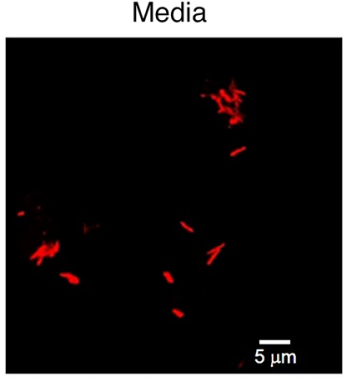

C
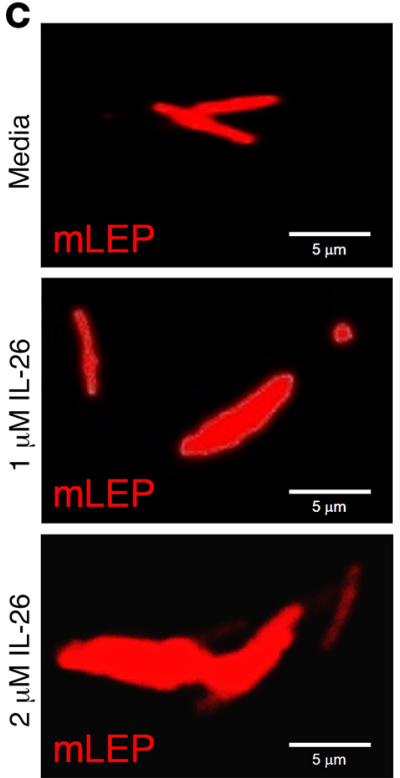

1 uM IL-26
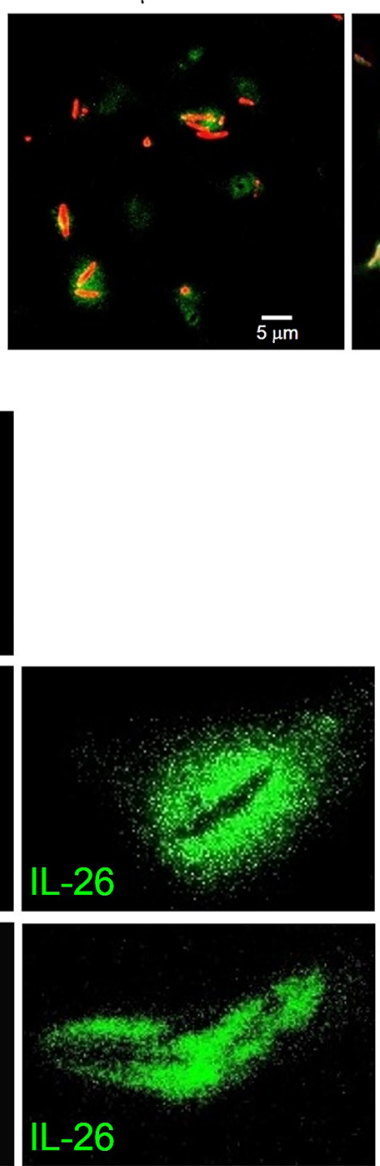

2 uM IL-26
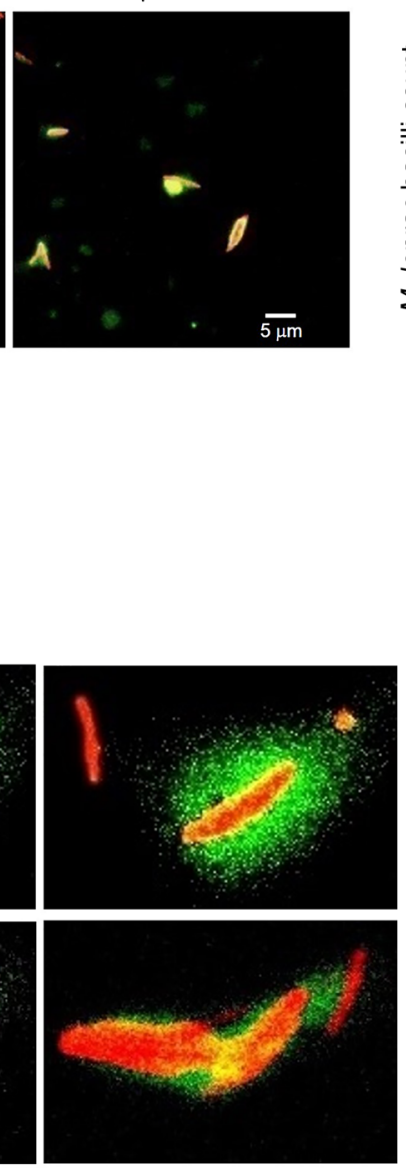

B Axenic culture

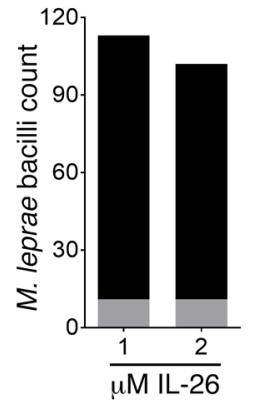

Axenic culture

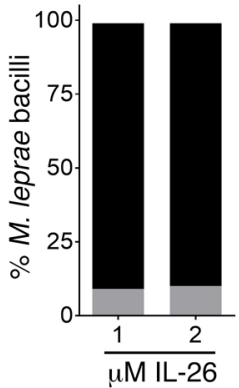

Bound Free

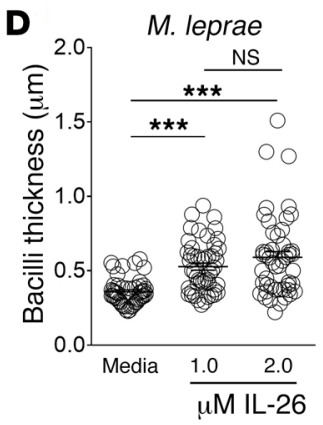

Figure 4. IL-26 binds directly to M. leprae bacilli. (A and C) Confocal microscopic images of Alexa 488-IL-26 (green) cultured with M. leprae (red) for 6 hours. Data shown are representative of 4 independent experiments. Scale bars: $5 \mu \mathrm{m}$. (B) Quantification of number of free bacilli or IL-26-bound bacilli (left) and percentage of free or IL-26-bound bacilli of total bacilli counts (right). (D) Measurement of M. leprae bacilli thickness from the confocal microscopy images in C. Media contained Alexa Fluor 488 dye as a control. Data represent the mean \pm SEM ( $n=50$ bacilli for each condition). Data shown are representative of 4 independent experiments. ${ }^{* *} P<0.001$, by repeated-measures 1 -way ANOVA.

IL-26 induces antimicrobial activity against intracellular bacteria. Finally, we sought to determine whether IL-26 treatment during infection would result in antimicrobial activity against $M$. leprae. Indeed, we found that IL-26 treatment during $M$. leprae infection significantly reduced the viability of intracellular bacteria compared with media control. The addition of $2 \mu \mathrm{M}$ IL-26 to the infected MDMs was required to reduce the viability of the intracellular bacteria by approximately $40 \%$, much less than the $10-\mu \mathrm{M}$ amount needed to have similar effects in axenic culture $(P$ $<0.05$, Figure 8A). Antimicrobial activity was dependent on the native structure of IL-26, because denaturing the protein did not produce the same results $(P<0.05$, Figure $8 \mathrm{~B})$. We observed similar antimicrobial activity against $M$. tuberculosis (H37Ra) in MDMs $(P<0.05$, Figure 8C). We note that in one study, the addition of IL-26 to M. tuberculosis-infected whole blood, albeit at lower concentrations ( 700 -fold lower than given here), did not result in an antimicrobial response; however, the authors stated that the nature of the whole-blood antimicrobial assay made interpretation of this result difficult (11).

To further investigate the ability of IL-26 to mediate an antimicrobial response against intracellular bacteria, we infected
MDMs with Staphylococcus aureus, which, when taken up by macrophages, has been reported to inhibit autophagy and acidification of phagolysosomes (32-34). Compared with the media control, we observed that IL-26 treatment of MDMs significantly reduced the viability of intracellular $S$. aureus $(P<0.05$, Figure $8 \mathrm{D})$. Having demonstrated that IL-26 can induce autophagy in MDMs, we sought to determine whether autophagy is required for antimicrobial activity. We used the PI3K inhibitor wortmannin to block IL-26-induced autophagy. The addition of wortmannin prior to IL-26 stimulation significantly reduced antimicrobial activity, which we observed by the increased viability of $M$. leprae, from $53 \% \pm 7.8 \%$ with $2 \mathrm{M}$ IL-26 treatment to $86.7 \% \pm 10.1 \%$ with wortmannin $(P<0.05$, Figure $8 \mathrm{E})$. In summary, these data demonstrate that IL-26 treatment of infected MDMs leads to autophagy, colocalization of IL-26 with the bacilli in LAMP1 compartments, and an antimicrobial response against intracellular bacteria.

\section{Discussion}

The adaptive $\mathrm{T}$ cell response kills intracellular pathogens through direct and indirect mechanisms. For example, $\mathrm{CD}^{+}$cytotoxic $\mathrm{T}$ cells deliver cytotoxic granule contents including the antimicro- 

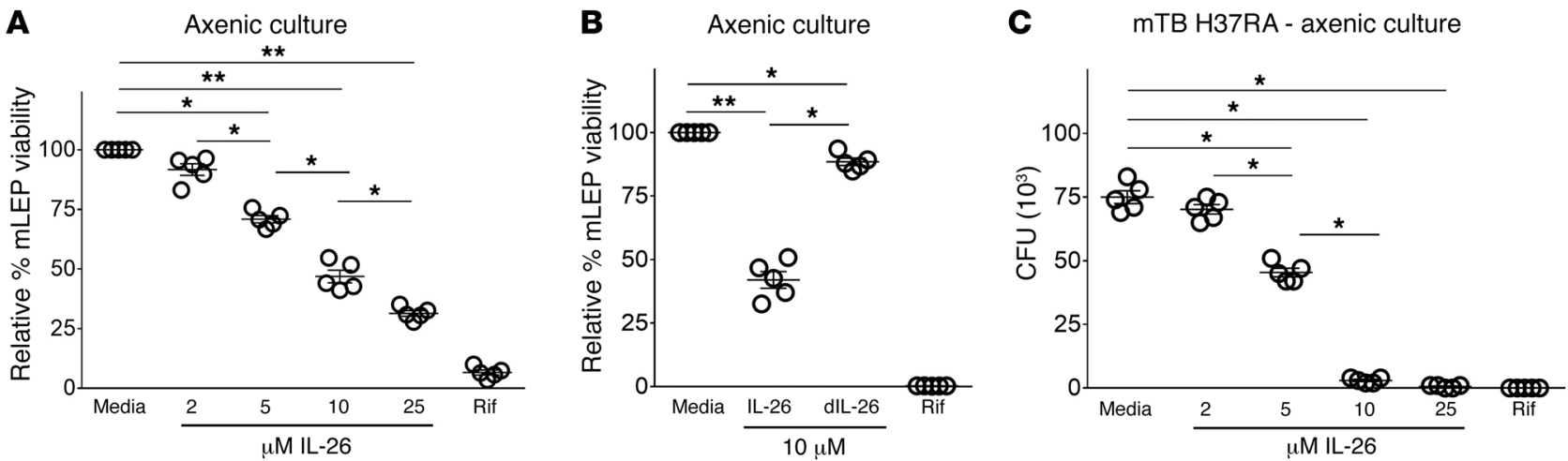

Figure 5. IL-26 has direct antimicrobial activity against mycobacteria. (A) M. leprae was cultured for 4 days with increasing concentrations of IL-26. Rifampicin (Rif) was used as a positive control. Viability of $M$. leprae was calculated by the ratio of bacterial 16S rRNA and RLEP DNA detected by qPCR. Relative viability was determined by comparing the treatment ratio with the media ratio. Data represent the mean \pm SEM $(n=5)$. (B) M. leprae (mLEP) was cultured for 4 days with $10 \mu \mathrm{M}$ native IL-26 or denatured IL-26 (dIL-26). Rifampicin was used as a positive control. Viability of M. leprae was calculated according to the ratio of bacterial 165 rRNA and RLEP DNA detected by qPCR. Relative viability was determined by comparing the treatment ratio with the media ratio. Data represent the mean \pm SEM $(n=5)$. (C) M. tuberculosis (mTB H37Ra) was cultured for 4 days with increasing concentrations of IL-26. Rifampicin was used as a positive control. A CFU assay was performed and numbers quantified after 3 weeks. Data represent the mean \pm SEM $(n=5)$. ${ }^{*} P<0.05$ and ${ }^{* *} P<0.01$, by repeated-measures 1-way ANOVA.

bial protein granulysin into infected macrophages, leading to a direct reduction in the viability of intracellular pathogens (35-38). $\mathrm{CD}^{+}$Th1 cells release IFN- $\gamma$, which triggers an indirect antimicrobial mechanism through activation of endogenous pathways in macrophage microbicidal pathways. Given that $\mathrm{CD}^{+}$Th17 cell production of IL-26 provides a direct antimicrobial pathway against extracellular bacteria (9), we explored whether IL-26 can mediate an antimicrobial response against intracellular bacteria. We provide evidence that IL-26 enters bacteria-infected macrophages, induces autophagy, and colocalizes with the pathogen, together resulting in an antimicrobial response. These data identify a role for IL-26 in host defense against intracellular bacteria.

We examined the role of IL-26 in leprosy, which provides a unique model, given that the disease presents as a spectrum in which the clinical presentation correlates with the immune response to the pathogen. Measuring mRNA and protein levels at the site of disease, we observed that IL-26 expression was greater in lesions from patients with T-lep than in those with L-lep, correlating with the group of patients with the greatest cell-mediated immunity against the pathogen. IL-26 colocalized with T cells but also with $\mathrm{CD} 68^{+}$macrophages, indicating proximity to the cell type infected by the leprosy bacillus. The colocalization of IL-26 with $\mathrm{CD}^{+} 8^{+}$macrophages was significantly $(P<0.05)$ greater in T-lep than L-lep lesions, even though there are comparable numbers of macrophages in the different forms of leprosy (38). The higher expression levels of IL-26 in T-lep lesions correlate with the lower bacterial load in this form of leprosy $(39,40)$. In contrast, the relatively lower expression of IL-26 that we observed in L-lep lesions is associated with a higher bacterial load in this form of leprosy. The lack of IL-26 may permit bacterial growth, and/or the bacilli may potentially inhibit the production and secretion of IL-26. For example, expression of IL-4, IL-10, and type I IFN has been reported to be higher in L-lep compared with T-lep lesions, and these cytokines are known to inhibit antimycobacterial pathways $(22,41-44)$. Accordingly, the type IIFN gene expression profile is also enhanced in the blood of patients with active tuberculosis $(45,46)$, which may contribute to the lower amount of IL-26 release following $M$. tuberculosis infection of a monocyte-enriched population from patients with tuberculosis compared with healthy controls (11).

IL-26 may contribute to an antimicrobial response against intracellular bacteria in infected macrophages through 2 mechanisms. First, IL-26 had direct antimicrobial activity against intracellular mycobacteria, as shown in axenic culture, and colocalized with $M$. leprae in infected MDMs. Second, we found that IL-26 induces autophagy in MDMs, which was required for its antimicrobial response against intracellular $M$. leprae. Since mycobacteria are known to inhibit the phagolysosomal fusion required for intracellular killing (47), and S. aureus is known to subvert acidification of phagolysosomes (32-34), IL-26 may stimulate infected cells to overcome this inhibition, facilitating the antimicrobial response. Additionally, the higher expression of IL-26 we detected in T-lep lesions also correlates with the higher number of autophagosomes detected in this form of leprosy (48). Although autophagy is required for killing of intracellular mycobacteria, some intracellular bacteria evade autophagy-dependent killing (28-30).

We found that IL-26-induced autophagy was dependent on the cytoplasmic DNA receptor STING, consistent with the demonstrated ability of IL-26 to bind to DNA from dying cells in in vitro cultures and traffic this DNA to activate STING $(9,31)$, and the ability of STING activation to trigger autophagy in mycobacteriainfected macrophages (49). Although the IL-26 receptor, a heterodimer composed of IL-10R2 and IL-20R1, is expressed exclusively and constitutively by epithelial cells $(15,16)$, human monocytes and macrophages express IL-10R2 but not IL-20R1. One report demonstrated that IL-26 induction of IL-6 from monocytes was blocked by approximately $50 \%$ using a polyclonal goat antiIL-10R2 Ab, thereby triggering IL-10R2 alone or in combination with an undetermined coreceptor (19). It is therefore possible that IL-10R2 in myeloid cells is involved in uptake and/or signaling of IL-26 to induce autophagy.

Given that IL-26 is produced by Th17 cells, our data, along with previous studies, suggest the possibility that Th17 cells con- 
A
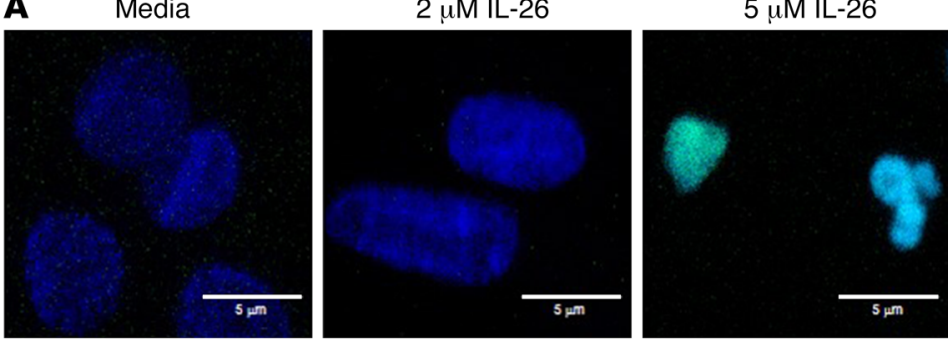

$1 \mu \mathrm{M} \mathrm{IL}-26$

B

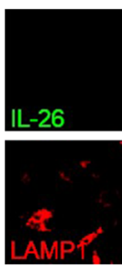

Media
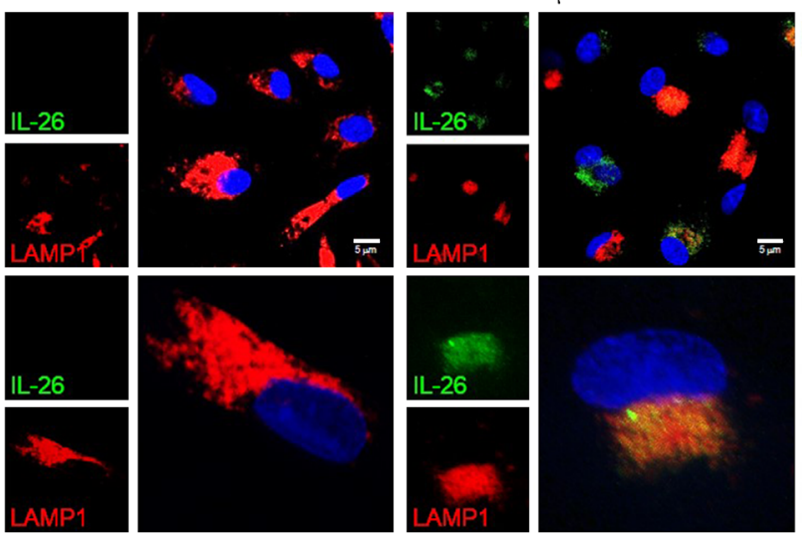

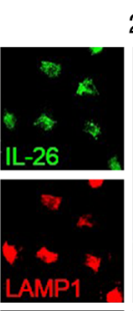

$2 \mu \mathrm{M}$ IL-26

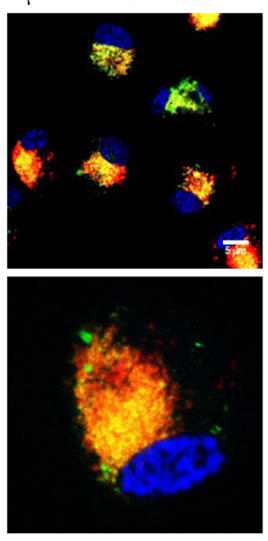

Act. D positive ctrl
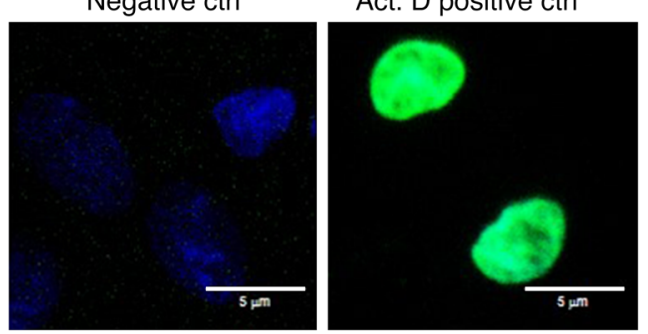

C

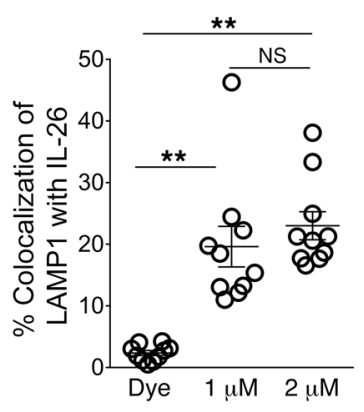

D
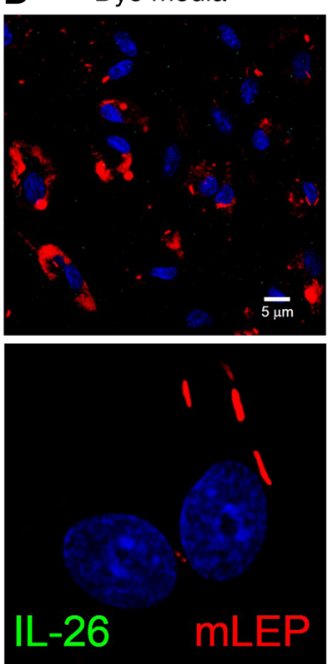

1 uM IL-26
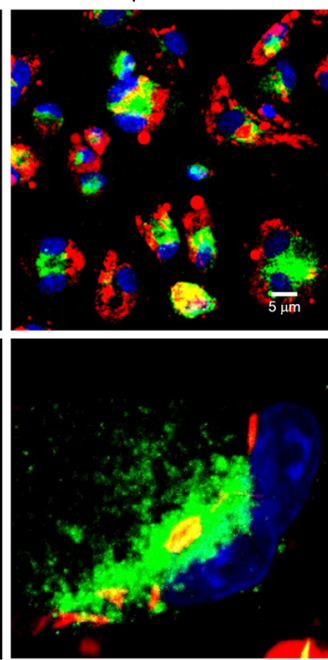

$2 u \mathrm{M}$ IL-26

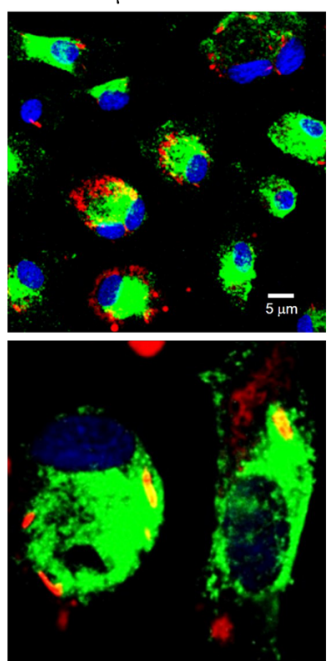

E M. leprae-infected MDMs

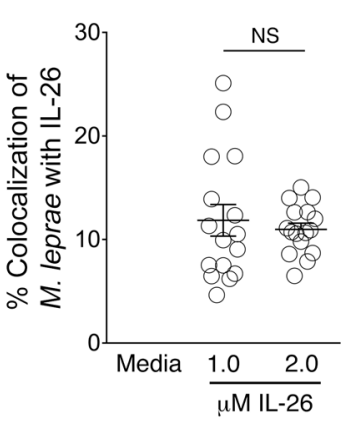

F IL-26-pretreated MDMs

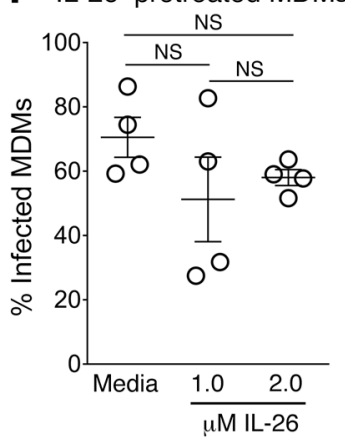

Figure 6. IL-26 is taken up by MDMs and colocalizes with M. leprae. (A) Human MDMs were treated with IL-26 overnight. Cells were washed and fixed, and apoptosis was determined using a TUNEL (green) assay. Nuclei were stained with DAPI (blue). Data shown are from 5 individual donors. Scale bars: $5 \mu$ m. (B) Human MDMs were treated with Alexa 488-IL-26 (green) overnight. Cells were washed, fixed, and immunolabeled with anti-LAMP1 Ab (red). Nuclei were stained with DAPI (blue). Data shown are representative of 5 individual donors. Scale bars: $5 \mu \mathrm{m}$. Bottom row magnification, $\times 630$. (C) Colocalization of LAMP1 (red) and IL-26 (green) was quantified with Image). Data represent the mean percentage of colocalization \pm SEM ( $n \geq 50$ cells from 3 donors). ${ }^{*} P<0.01$, by repeated-measures 1-way ANOVA. (D) Human MDMs were treated with Alexa 488-IL-26 (green) for 30 minutes and infected with M. leprae (red) overnight. Cells were washed and fixed. Nuclei were stained with DAPI (blue). Media contained Alexa Fluor 488 dye as a control. Data shown are from 4 individual donors. Scale bars: $5 \mu \mathrm{m}$. Bottom row magnification, $\times 630$. (E) Colocalization of M. leprae (red) and IL-26 (green) was quantified with Image). Data represent the mean percentage of colocalization \pm SEM ( $n \geq 40$ cells from 4 donors). (F) Quantification of M. leprae-infected MDMs following 30 minutes of treatment with IL-26. Data represent the mean percentage $\pm \operatorname{SEM}(n=4)$.

tribute to host defense in leprosy. Serum IL-17 was lowest in L-lep patients compared with serum levels in all other forms of leprosy (50). Th17 cells were more frequent in both the lesions and $M$. leprae-stimulated PBMCs in patients with T-lep versus those with L-lep (51). Furthermore, SNPs in $I L 17 F$ were associated with lepro- sy susceptibility (52). The related bacterium M. tuberculosis induces IL-23, a key cytokine in Th17 polarization by DCs (53), as well as Th17 cells (54). Yet, $I L-17 R^{-/-}$(55) and $I L-23^{-/-}$(54) mice were more susceptible to M. tuberculosis infection. In mouse models of tuberculosis, IL-17 was required for the generation of IFN- $\gamma$-producing 

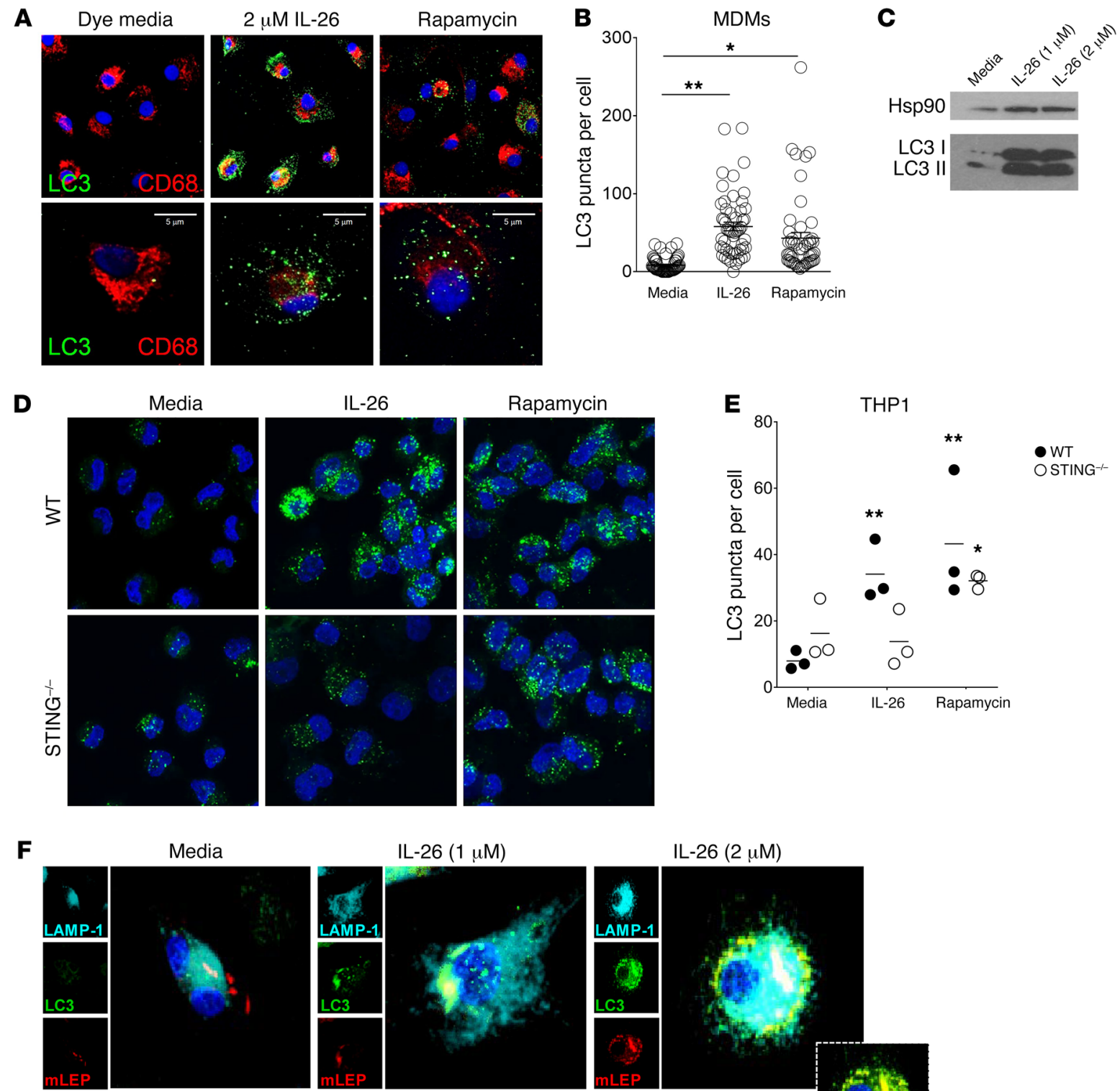

IL-26 (2 $\mu \mathrm{M})$

G

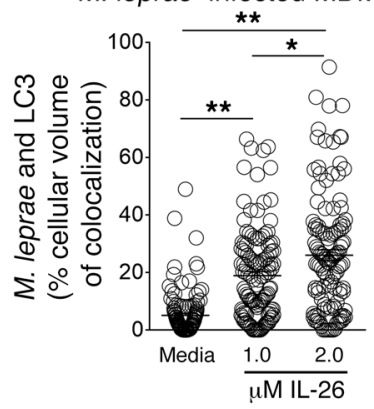

I

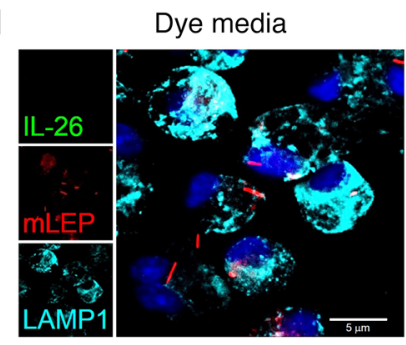

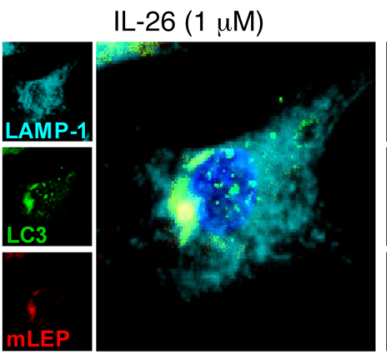

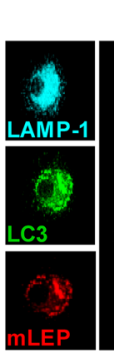

H M. leprae-infected MDMs
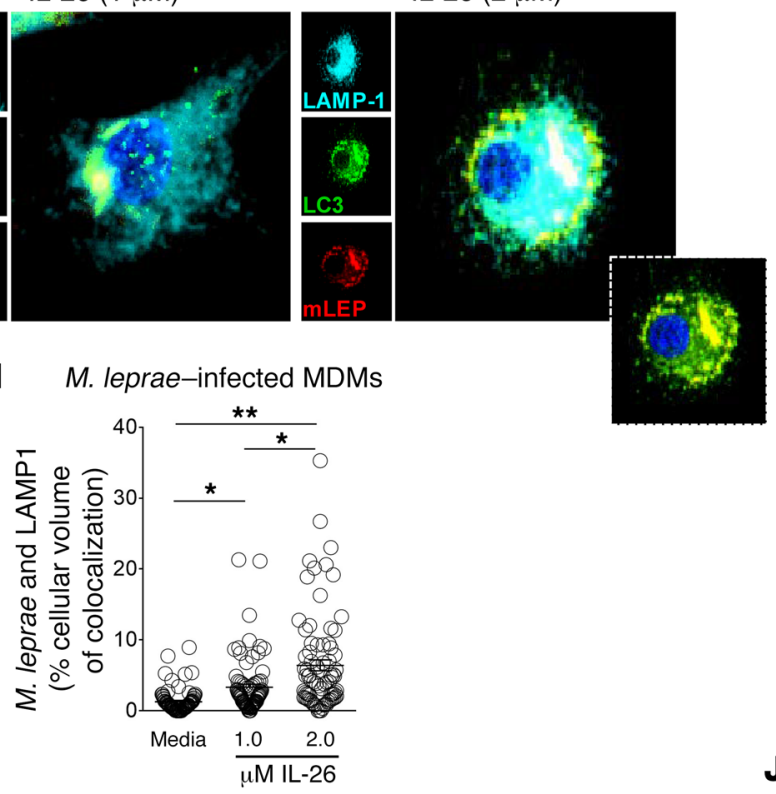

J M. leprae-infected MDMs

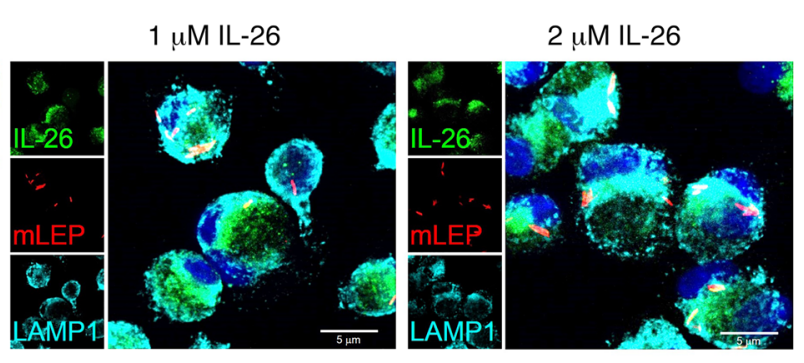

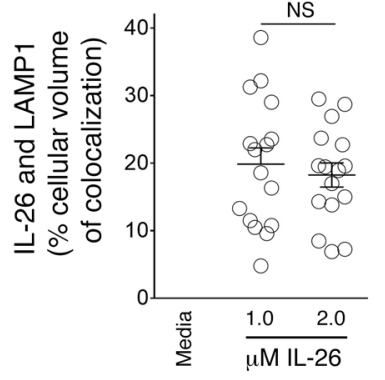


Figure 7. IL-26 induces autophagy and enhances bacterial trafficking to the lysosomes. (A) MDMs were cultured with IL-26 or media overnight and then immunolabeled with anti-LC3 Ab (green) and anti-CD68 Ab (red). Nuclei were stained with DAPI (blue). Original magnification, $x 63$. Scale bars: $5 \mu \mathrm{m}$. (B) LC3 puncta per cell \pm SEM ( $n \geq 50$ cells from 4 donors). (C) LC3 I to LC3 II conversion was detected by immunoblotting. Hsp90 was used as an internal control. (D) PMA-treated THP-1 cells were treated with IL-26 $(2 \mu \mathrm{M})$ for 24 hours and with rapamycin $(300 \mathrm{nM})$ for 6 hours and then immunolabeled with LC3 Ab (green). Nuclei were stained with DAPI (blue). Data shown are representative of 1 of 3 independent experiments for both WT and STING ${ }^{-1-}$ THP-1 cells. Original magnification, $\times 63$. (E) LC3 puncta per cell \pm SEM ( $n \geq 50$ cells from 3 donors for both WT and STING ${ }^{-1-}$ THP-1 cells). (F) Human MDMs were treated with IL-26 for 30 minutes and infected with $M$. leprae (red) overnight. Cells were washed, fixed, and immunolabeled with anti-LC3 (green) and LAMP-1 (cyan). Nuclei were stained with DAPI (blue). Data shown are representative of 4 individual donors. Inset image is of LC3 (green) and M. leprae (red) overlay, without LAMP1 (cyan). Original magnification, $\times 63$ and x630 (enlarged insets). (C) Colocalization of LC3 and M. leprae and (H) colocalization of LAMP1 and M. leprae were quantified with ImageJ. Data represent the mean percentage of the cellular volume of colocalization \pm SEM ( $n \geq 30$ cells from 4 donors). (I) Human MDMs were treated with Alexa 488-IL-26 (green) for 30 minutes and infected with M. leprae (red) overnight. Cells were washed and immunolabeled with anti-LAMP1 (cyan). Nuclei were stained with DAPI (blue). Media contained Alexa Fluor 488 dye as a control. Data shown are from 4 individual donors. Scale bars: $5 \mu \mathrm{m}$. (J) Colocalization of IL-26 and LAMP1 was quantified with ImageJ. Data are represented as the mean percentage of the cellular volume of colocalization $\pm \operatorname{SEM~(~} n \geq 40$ cells from 4 donors). ${ }^{*} P<0.05$ and ${ }^{* *} P<0.01$, by repeated-measures 1-way ANOVA.

Th1 cells (56), granuloma formation, inflammation $(54,56)$, and vaccine-induced immunity (57). However, the functional studies of Th17 cells in tuberculosis have utilized mouse models, and, as mentioned, IL-26 is not present in the mouse genome, such that it will be important to determine whether Th17 cells contribute to host defense through mechanisms other than IL-26 release.

In addition to mycobacteria, our data indicate that IL-26 has an antimicrobial effect on another cutaneous pathogen, $S$. aureus, when taken up by macrophages. Previous studies reported that defective Th17 responses in STAT3-deficient patients have been associated with increased susceptibility to $S$. aureus and Streptococcus pyogenes infections $(58,59)$, potentially due to reduced STAT3 activation by IL-26 (60).

The current immunologic premise holds that Th1 cells defend against intracellular pathogens, whereas Th17 cells are required to defend against extracellular bacteria. In previous work, we demonstrated that Th1 cell release of IFN- $\gamma$ activates macrophages to kill intracellular mycobacteria $(22,30)$, while human Th17 cells release IL-26, which kills extracellular bacteria in axenic (i.e., cellfree) cultures (9). Nevertheless, vaccines that induce only Th1 cells are not sufficient to engender protection against intracellular mycobacteria (61). Our data provide evidence that the Th17 cytokine IL-26 can contribute to host defense against intracellular bacteria, identifying one mechanism by which Th17 cells contribute to host defense against such pathogens.

\section{Methods}

Microarray data analysis. The reported gene expression profiles of mRNAs derived from skin biopsy specimens from 16 patients with leprosy (T-lep, $n=10$; L-lep, $n=6$ ), as determined using Affymetrix
Human U133 Plus 2.0 microarrays, were mined to assess IL26 mRNA expression $(22,62)$. The gene expression files containing these array data are available in the NCBI's Gene Expression Omnibus (GEO) database (GEO GSE17763).

Generation of MDMs. MDMs were derived from whole blood from healthy donors. PBMCs were isolated using Ficoll (GE Healthcare) gradient centrifugation. $\mathrm{CD}^{4} 4^{+}$cells were positively selected from PBMCs using CD14 MicroBeads (Miltenyi Biotec, 130-050-201) and cultured in the presence of macrophage colony-stimulating factor (M-CSF) $(50 \mathrm{ng} / \mathrm{ml})$ (R\&D Systems) and used on day 5 or 6 . We routinely achieved a greater than $90 \%$ purity of monocytes by CD14 immunomagnetic selection.

Viability of $M$. leprae by $q P C R$. Cells were stimulated with various concentrations of IL-26 for30 minutes and theninfected with M.leprae. For autophagy inhibition, MDMs were treated with $500 \mathrm{nM}$ wortmannin prior to IL-26 stimulation. mRNA was isolated from cells using TRIzol Reagent (Invitrogen Life Technologies, Thermo Fisher Scientific) according to the manufacturer's recommended protocol. The viability of intracellular $M$. leprae was determined by qPCR and quantified as previously described $(19,22,23)$. Briefly, comparison of the bacterial DNA with the mammalian 36B4 levels was used to monitor infectivity between all the conditions in the assay as well as PCR quality. The $16 \mathrm{~S} \mathrm{rRNA}$ and genomic DNA values were calculated using the $\Delta \Delta \mathrm{Ct}$ analysis, with the bacterial DNA value serving as the housekeeping gene. The primer sequences were as follows: M. leprae 16s RNA, forward, 5'-GCATGTCTTGTGGTGGAAAGC-3'; $M$. leprae 16s RNA, reverse, 5'-CACCCCACCAACAAGCTGAT-3'; M. leprae RLEP DNA, forward, 5'-GCAGCAGTATCGTGTTAGTGAA-3'; M. leprae RLEP DNA, reverse, 5'-CGCTAGAAGGTTGCCGTAT-3'; H36B4, forward, 5'-CCACGCTGCTGAACATGCT-3'; H36B4, reverse, 5'-TCGAACACCTGCTGGATGAC-3'.

M. tuberculosis axenic cultures. Recombinant IL-26 monomers (R\&D Systems) were diluted in $10 \mathrm{mM}$ sodium phosphate, $\mathrm{pH}$ 7.2. Aliquots of $2.5 \times 10^{5} \mathrm{M}$. tuberculosis (H37Ra) bacteria were added to 100 $\mu \mathrm{l}$ Middlebrook 7H9 medium (BD Biosciences) containing recombinant IL-26 monomers (R\&D Systems) or $20 \mu \mathrm{g} / \mathrm{ml}$ (final concentration) rifampicin (Sigma-Aldrich). Cultures were incubated at $37^{\circ} \mathrm{C}$ for 72 hours, after which $900 \mu \mathrm{l}$ of $10 \mathrm{mM}$ sodium phosphate, $\mathrm{pH} 7.2$, was added to attain a volume of $1 \mathrm{ml}$. From the 1-ml suspension, $10 \mu \mathrm{l}$ was plated onto Middlebrook 7H10 agar plates (Teknova) for 3 weeks, and CFU were enumerated.

Infection of MDMs and quantification of M. tuberculosis CFU. Human MDMs were pretreated with IL-26 (R\&D Systems) or $1 \mu \mathrm{g} / \mathrm{ml}$ (final concentration) rifampicin (Sigma-Aldrich) in 10\% Omega FCS for 30 minutes and infected with M. tuberculosis (H37Ra) at a MOI of 5. The infected cells were harvested after 4 days, pelleted, and lysed with $100 \mu \mathrm{l}$ of $0.2 \%$ saponin in $1 \times$ PBS on ice for 20 minutes. PBS $1 \times$ $(900 \mu \mathrm{l})$ was added to bring the cell lysate to a 1-ml final volume. From the 1-ml suspension, $1 \mu \mathrm{l}$ stock was plated onto Middlebrook $7 \mathrm{H} 10$ agar plates (Teknova) for 3 weeks, and CFU were enumerated.

IL-26 culture with M. leprae. Recombinant IL-26 monomers (R\&D Systems) were diluted in $10 \mathrm{mM}$ sodium phosphate, $\mathrm{pH} 7.2$, and labeled using the Alexa Fluor 488 Microscale Protein Labeling Kit (Molecular Probes, Invitrogen, Thermo Fisher Scientific). Aliquots of $1 \times 10^{6}$ M. leprae bacteria, labeled by PKH-26, were incubated with A488IL-26 in a 50- $\mu \mathrm{l}$ volume of $10 \mathrm{mM}$ sodium phosphate, $\mathrm{pH} 7.2$, for 6 hours and then mounted onto microscopy slides with ProLong Gold (Invitrogen Life Technologies, Thermo Fisher Scientific). Images were 

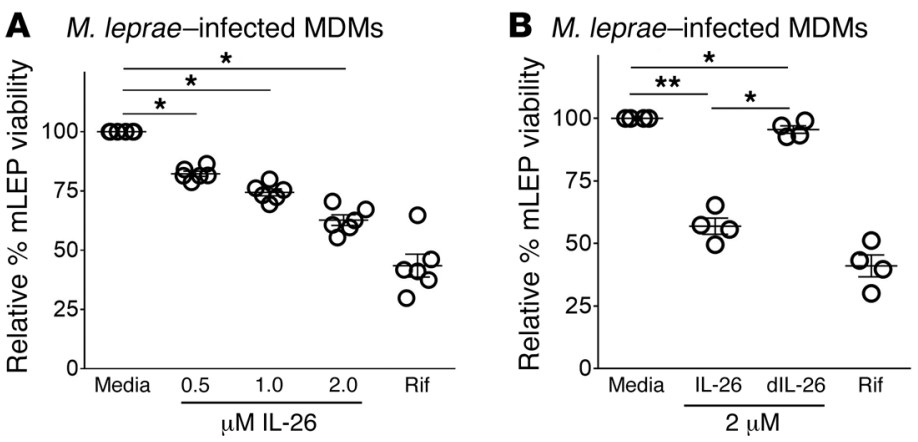

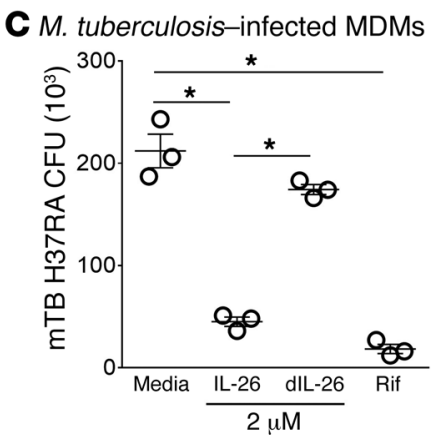

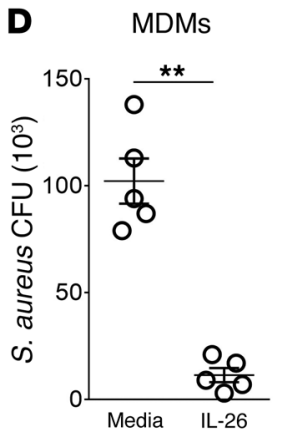

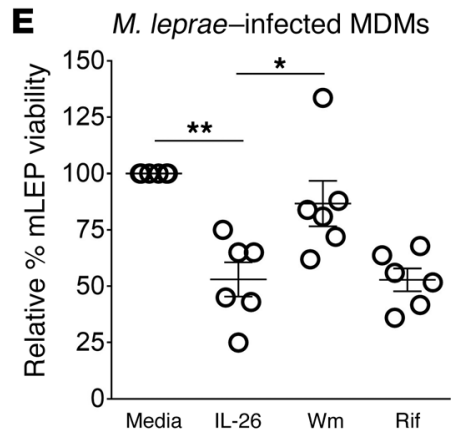

Figure 8. IL-26 induces antimicrobial activity against intracellular bacteria. (A) MDMs were treated with IL-26 for 30 minutes and then infected with M. leprae for 4 days. Rifampicin was used as a positive control. Viability of $M$. leprae was measured by qPCR $(n=6)$. (B) MDMs were treated with IL-26 or denatured IL-26 and then infected with $M$. leprae, and bacterial viability was measured as in A $(n=4)$. (C) MDMs were treated with IL-26 or denatured IL-26 and then infected with M. tuberculosis (H37Ra) as in A. Viability of intracellular M. tuberculosis was determined by CFU assay $(n=3)$. (D) MDMs were treated with IL-26 for 4 hours and then washed and infected with S. aureus for 4 hours, followed by removal of the extracellular bacteria. After overnight culture, the viability of intracellular $S$. aureus was determined by CFU assay $(n=5)$. (E) MDMs were treated with IL-26 with or without wortmannin $(\mathrm{Wm})$ and then infected with $M$. leprae, and bacterial viability was measured as in $\mathbf{A}(n=6)$. ${ }^{*} P<0.05$ and ${ }^{* *} P$ $<0.01$, by repeated-measures 1-way ANOVA. All data represent the mean \pm SEM.

examined using a Leica microscope. $M$. leprae bacilli diameters were measured using Imaris software.

Infection of MDMs and quantification of $M$. leprae viability. $M$. leprae bacteria were grown in the footpads of nu/nu mice as described previously (63) and were provided by the National Hansen's Disease Program. Human MDMs were pretreated with IL-26 (R\&D Systems) in $10 \%$ Omega FCS for 30 minutes and infected with $M$. leprae at a MOI of 5. The infected cells were harvested after 4 days. RNA and DNA were isolated from infected cells using TRIzol (Invitrogen, Life Technologies, Thermo Fisher Scientific). cDNA was made from RNA. DNA was treated with RNase to removed residual RNA. The viability of intracellular M. leprae was determined by qPCR and quantified as previously described $(22,24)$. Briefly, the levels of bacterial $16 \mathrm{~S}$ rRNA and the genomic DNA element RLEP of M. leprae were measured by qPCR. The 16S rRNA and RLEP DNA values were determined by $\Delta \Delta \mathrm{Ct}$ analysis, with the DNA value serving as the housekeeping gene. The ratio of RNA to DNA was calculated for each replicate, and the percentage of bacterial viability was calculated relative to the respective media control. The efficiency of infection was determined by confocal microscopy. Approximately $5 \%$ to $70 \%$ of the cells were infected. Of the total number of cells, more than $95 \%$ were viable following the washes and infection. To denature recombinant IL-26, the protein was treated with $5 \% \beta$-mercaptoethanol (Invitrogen, Thermo Fisher Scientific) and heated at $95^{\circ} \mathrm{C}$ for 10 minutes.

Infection of MDMs and quantification of intracellular $S$. aureus $C F U$. Bacteria of the S. aureus strain DU5938 ( $\mathrm{Hla}^{-} \mathrm{Hlb}^{-} \mathrm{Hlg}^{-}$) (64) were grown to the mid-log phase at $37^{\circ} \mathrm{C}$ with shaking (150 rpm) in brainheart infusion (BHI) medium and then collected by centrifugation for 10 minutes at $5000 \mathrm{~g}$. For cellular infection, the bacterial suspension was diluted with sterile $1 \times$ PBS. The number of viable bacteria was determined by serial dilution and plating onto BHI agar plates (65). Human MDMs $\left(2 \times 10^{6}\right.$ cells $)$ were pretreated with IL-26 for 4 hours, washed, and infected with $S$. aureus at an MOI of 5 for 4 hours in RPMI with $10 \%$ FCS antibiotic-free media. Next, $100 \mu \mathrm{g} / \mathrm{ml}$ gentamycin was added to the wells for 20 minutes to kill extracellular bacteria. Cells were then washed to further remove any remaining extracellular bacteria. Infected cells were cultured overnight at $37^{\circ} \mathrm{C}$ in a $4 \% \mathrm{CO}_{2}$ incubator. To measure intracellular killing of bacteria, cells were pelleted and lysed with $100 \mu \mathrm{l}$ of $0.2 \%$ saponin in $1 \times$ PBS on ice for 20 minutes. Next, $1 \times$ PBS $(900 \mu \mathrm{l})$ was added to bring the cell lysate to a final volume of $1 \mathrm{ml}$, of which $1 \mu \mathrm{l}$ was plated and CFU determined.

Patients and clinical specimens. Patients with leprosy were classified according to the criteria established by Ridley and Jopling (66). The designation of T-lep included patients who were classified clinically as borderline tuberculoid ("BT"), and the designation of L-lep only included patients classified as "LL." All T-lep and L-lep skin biopsy specimens were taken at the time of diagnosis, prior to the initiation of treatment. Specimens were embedded in OCT medium (Ames), snap-frozen in liquid nitrogen, and stored at $-80^{\circ} \mathrm{C}$.

Tissue immunoperoxidase labeling. Frozen tissue sections were blocked with normal horse serum before incubation 2 hours with mAbs against IL-26 (clone 2A8, IgG2a, Sigma-Aldrich) and CD3 (clone UCHT1, IgG1k, BD Biosciences), followed by incubation with biotinylated horse anti-mouse IgG for 90 minutes. To test Ab specificity, IL-26 Ab was incubated with IL-26 monomers (R\&D Systems) for 15 minutes prior to incubation on tissue sections. Slides were counterstained with hematoxylin and mounted in crystal mounting medium (Biomeda) and were visualized using the ABC Elite system (Vector Laboratories). Skin sections were examined with a Leica microscope. Ratios were calculated using ImmunoRatio online software (Jorma Isola and Vilppu Tuominen, Institute of Biomedical Technology, University of Tampere, Tampere, Finland) (23), an automated image analysis application that calculates the percentage of DAB-stained nuclear area per total area.

Cell culture immunofluorescence labeling. Recombinant IL-26 monomers (R\&D Systems) were labeled using the Alexa Fluor 488 
Microscale Protein Labeling Kit (Molecular Probes, Invitrogen, Thermo Fisher Scientific). MDMs were treated with labeled recombinant IL-26 (rIL-26) monomers in RPMI media with 10\% FCS for 30 minutes. As a control, MDMs were treated with the Alexa Fluor 488 dye media used for the labeling reaction. Treated MDMs were next infected with live PKH26-labeled M. leprae overnight. Cells were then washed and fixed for 30 minutes with $4 \%$ PFA and then washed again. Next, cells were permeabilized with $0.25 \%$ saponin for 20 minutes, blocked with serum for 30 minutes, and stained with primary Abs against CD68 (clone Y1/82A, IgG2b, BioLegend), LAMP1 (clone H4A3, IgG1, BioLegend), or LC3 (clone 4E12, IgG1, MBL International) for 2 hours. Following washing, cells were stained with secondary Abs (Molecular Probes, Invitrogen, Thermo Fisher Scientific) for 90 minutes, washed, and mounted with ProLong Gold with DAPI (Invitrogen, Life Technologies, Thermo Fisher Scientific). For the quantification of autophagy, the percentages of LC3 punctated cells were evaluated using fluorescence microscopy. Approximately 100 cells, over 6 different random fields of view, were scored for each condition of each experiment. Rapamycin was used as a positive control for autophagy induction at $300 \mathrm{nM}$. Immunofluorescence of cell cultures was examined using a Leica TCS-SP MP inverted single confocal laser-scanning microscope and a Leica 2-photon laser microscope at the Advanced Microscopy/Spectroscopy Laboratory and Macro-Scale Imaging Laboratory (California NanoSystems Institute, UCLA). Colocalization quantifications were performed using ImageJ software (NIH) by splitting the final image overlay into individual single channels and selecting the "Colocalization Threshold" option to calculate the percentage of pixel overlap between channels.

Culture and treatment of WT and STING-KO THP-1 cells. WT and STING-KO THP-1 dual reporter cell lines were purchased from InvivoGen. The STING-KO THP-1 dual cells were created by stable KO of STING in WT THP-1 dual cells. The cells were cultured according to the manufacture's protocol. For all experiments, both WT and STINGKO THP- 1 cells were passaged no more than 5 times. WT and STINGKO cells were plated at a density of $0.5 \times 10^{6}$ cells $/ \mathrm{ml}$ on 8 -well glass slides and were made adherent by treating with $400 \mathrm{ng} / \mathrm{ml}$ PMA for 48 hours. The cells were subsequently treated or not with $2 \mu \mathrm{M}$ rIL26 monomers for 24 hours. Rapamycin $(300 \mathrm{nM})$ was added to some wells for 3 hours to serve as a positive control for autophagy induction. Immunofluorescence labeling for LC3 puncta detection was performed as described above in the Cell culture immunofluorescence labeling section, with quantification of the number of LC3 puncta per cell done using Image J software.

Tissue immunofluorescence labeling. Immunofluorescence was performed by serial incubation of cryostat tissue sections with anti-human mAbs of different isotypes for 2 hours (IL-26, clone 2A8, IgG2a, Sigma-Aldrich; CD4, clone A16A1, IgG2b, BioLegend; CD8, clone HIT8a, IgG1, BioLegend; CD68, clone KP1, IgG1, Abcam), and then washed 3 times with $1 \times$ PBS, followed by incubation with isotype-specific fluorochrome A488-, A568-, and A647-labeled goat anti-mouse Ig Abs (Molecular Probes, Invitrogen, Thermo Fisher Scientific) for 90 minutes. Negative controls were stained with matching isotype Abs. Nuclei were stained with DAPI (Invitrogen, Life Technologies, Thermo Fisher Scientific). Immunofluorescence of skin sections was examined using a Leica TCS-SP MP inverted single confocal laser-scanning microscope and a 2-photon laser microscope (Leica) at the Advanced Microscopy/Spectroscopy and Macro-Scale Imaging Laboratory (Cal- ifornia NanoSystems Institute, UCLA). Colocalization quantifications were performed using ImageJ software.

Immunofluorescence colocalization analysis. The colocalization threshold plugin by ImageJ was used for all colocalization analyses related to confocal images. Briefly, this plugin compares 2 images for correlated pixel intensities, and a positive correlation between 2 images indicates that the signal in 1 channel (green) is observed at the same time as the signal in the other channel (red). The plugin performs a converging search for the image thresholds and reports various metrics on the resulting thresholded images. We used the percentage volume metric. The colocalization colors are: red + green = yellow; red + cyan = magenta; and red + green + cyan $=$ white .

TUNEL assay. To evaluate cell death by apoptosis, we used a Roche In Situ Cell Death Detection Kit with fluorescein according to the manufacturer's protocol. Briefly, human MDMs were differentiated on glass slides and treated with IL-26 or overnight. Cells were then washed, fixed, and labeled with TUNEL reaction mixture. Nuclei were stained with DAPI. Immunofluorescence was assessed using a Leica TCS-SP MP inverted single confocal laser-scanning microscope and a 2-photon laser microscope (Leica) at the Advanced Microscopy/Spectroscopy and Macro-Scale Imaging Laboratory (California NanoSystems Institute, UCLA).

Immunoblot analysis. MDMs $\left(2 \times 10^{6}\right.$ cells per condition $)$ were collected in NP-40 lysis buffer (50 mM Tris, pH 7.4, $50 \mathrm{mM} \mathrm{NaCl}, 5$ mM EDTA, $50 \mathrm{mM} \mathrm{NaF}, 1 \mathrm{mM} \mathrm{Na}_{3} \mathrm{VO}_{4}, 1 \% \mathrm{NP}-40,0.02 \% \mathrm{NaN}_{3}$, and $1 \mathrm{mM}$ PMSF) containing complete protease inhibitors (Roche Applied Science). Total protein from cell lysates was quantified by the Bradford assay. Lysates were separated on a 7.5\% nondenaturing gel, followed by immunoblot analysis with anti-HSP9O and anti-LC3, with detection by ECL (Pierce Biotechnology, Thermo Fisher Scientific).

Statistics. The statistics reported here cover the entire series of experiments and are described as the mean \pm SEM. GraphPad Prism 6 software was used for graphing and statistical analysis. For comparisons among 3 or more groups, we used repeated measures 1-way ANOVA with Greenhouse-Geisser correction, along with Tukey's multiple comparisons test, with individual variances computed for each comparison. A 2-tailed Student's $t$ test was used for all other 2-group analyses. For the WT and STING ${ }^{-/}$experiments, we used repeated-measures 2-way ANOVA, along with Tukey's multiple comparisons test. For all experiments, a $P$ value of less than 0.05 was considered statistically significant.

Study approval. This study was conducted according to the principles expressed in the Declaration of Helsinki. All donors provided written informed consent for the collection of peripheral blood and skin specimens for subsequent scientific analysis. All patients were recruited with approval from the IRBs of the USC School of Medicine and UCLA and the Institutional Ethics Committee of the Oswaldo Cruz Foundation.

\section{Author contributions}

RLM, ATD, RMBT and DIW designed the experiments, interpreted the data, and drafted the manuscript. BRB assisted with the conceptual framework and writing. ATD performed most of the experiments. DIW, KP, and GC performed the autophagy immunoblotting experiments. RMBT performed microarray analysis and quantified confocal imaging. ENS and MTO provided the clinical samples. MG assisted with the conceptual framework and writing of the manuscript. 


\section{Acknowledgments}

We would like to thank M. Schibler (California NanoSystems Institute at UCLA) and B. Andrade (Oswaldo Cruz Foundation), M. Fabri (University of Cologne), and S. Krutzik (David Geffen School of Medicine at UCLA) for their helpful discussions. The live M. leprae was provided by the US National Hansen's Disease Programs through the support of the American Leprosy Missions and Society of St.
Lazarus of Jerusalem. This work was supported in part by grants from the NIH (R01 AI022553, R01 AR040312, and P50 AR063020, to RLM) and the 2015 UCLA Dissertation Year Fellowship (to ATD).

Address correspondence to: Robert L. Modlin, UCLA Dermatology 52-121 CHS, 10833 Le Conte Avenue, Los Angeles, California, USA. Phone: 310.825.6214; Email: rmodlin@mednet.ucla.edu
1. Knappe A, Hör S, Wittmann S, Fickenscher $\mathrm{H}$. Induction of a novel cellular homolog of interleukin-10, AK155, by transformation of T lymphocytes with herpesvirus saimiri. J Virol. 2000;74(8):3881-3887.

2. Kotenko SV. The family of IL-10-related cytokines and their receptors: related, but to what extent? Cytokine Growth Factor Rev. 2002;13(3):223-240.

3. Stephen-Victor E, Fickenscher H, Bayry J. IL-26: an emerging proinflammatory member of the IL-10 cytokine family with multifaceted actions in antiviral, antimicrobial, and autoimmune responses. PLoS Pathog. 2016;12(6):e1005624.

4. Dumoutier L, Van Roost E, Ameye G, Michaux L, Renauld JC. IL-TIF/IL-22: genomic organization and mapping of the human and mouse genes. Genes Immun. 2000;1(8):488-494.

5. Donnelly RP, Sheikh F, Dickensheets H, Savan R, Young HA, Walter MR. Interleukin-26: an IL-10-related cytokine produced by Th17 cells. Cytokine Growth Factor Rev. 2010;21(5):393-401.

6. Wang T, Díaz-Rosales P, Martin SA, Secombes CJ Cloning of a novel interleukin (IL)-20-like gene in rainbow trout Oncorhynchus mykiss gives an insight into the evolution of the IL-10 family. Dev Comp Immunol. 2010;34(2):158-167.

7. Wilson NJ, et al. Development, cytokine profile and function of human interleukin 17-producing helper T cells. Nat Immunol. 2007;8(9):950-957.

8. Boniface $\mathrm{K}$, et al. Human Th17 cells comprise heterogeneous subsets including IFN-gammaproducing cells with distinct properties from the Th1 lineage. JImmunol. 2010;185(1):679-687.

9. Meller $\mathrm{S}$, et al. $\mathrm{T}(\mathrm{H}) 17$ cells promote microbial killing and innate immune sensing of DNA via interleukin 26. Nat Immunol. 2015;16(9):970-979.

10. Hughes T, et al. Stage 3 immature human natural killer cells found in secondary lymphoid tissue constitutively and selectively express the TH 17 cytokine interleukin-22. Blood. 2009;113(17):4008-4010.

11. Guerra-Laso JM, Raposo-García S, GarcíaGarcía S, Diez-Tascón C, Rivero-Lezcano OM. Microarray analysis of Mycobacterium tuberculosis-infected monocytes reveals IL26 as a new candidate gene for tuberculosis susceptibility. Immunology. 2015;144(2):291-301.

12. Che KF, et al. Interleukin-26 in antibacterial host defense of human lungs. Effects on neutrophil mobilization. Am J Respir Crit Care Med. 2014;190(9):1022-1031.

13. Zasloff M. Antimicrobial peptides of multicellular organisms. Nature. 2002;415(6870):389-395.

14. Ganz T. Defensins: antimicrobial peptides of innate immunity. Nat Rev Immunol. 2003;3(9):710-720.

15. Hör S, et al. The T-cell lymphokine interleukin-26 targets epithelial cells through the interleukin-20 receptor 1 and interleukin-10 receptor 2 chains. J Biol Chem. 2004;279(32):33343-33351.

16. Sheikh F, et al. Cutting edge: IL-26 signals through a novel receptor complex composed of IL-20 receptor 1 and IL-10 receptor 2.J Immunol. 2004;172(4):2006-2010.

17. Pène J, et al. Chronically inflamed human tissues are infiltrated by highly differentiated Th17 lymphocytes. JImmunol. 2008;180(11):7423-7430.

18. Miot C, et al. IL-26 is overexpressed in chronically HCV-infected patients and enhances TRAIL-mediated cytotoxicity and interferon production by human NK cells. Gut. 2015;64(9):1466-1475.

19. Corvaisier M, et al. IL-26 is overexpressed in rheumatoid arthritis and induces proinflammatory cytokine production and Th17 cell generation. PLoS Biol. 2012;10(9):e1001395.

20. Dambacher J, et al. The role of the novel Th17 cytokine IL-26 in intestinal inflammation. Gut. 2009;58(9):1207-1217.

21. Wilgus TA, Roy S, McDaniel JC. Neutrophils and wound repair: positive actions and negative reactions. Adv Wound Care (New Rochelle). 2013;2(7):379-388.

22. Teles RM, et al. Type I interferon suppresses type II interferon-triggered human anti-mycobacterial responses. Science. 2013;339(6126):1448-1453.

23. Tuominen VJ, Ruotoistenmäki S, Viitanen A, Jumppanen M, Isola J. ImmunoRatio: a publicly available web application for quantitative image analysis of estrogen receptor (ER), progesterone receptor (PR), and Ki-67. Breast Cancer Res. 2010;12(4):R56

24. Martinez AN, et al. Molecular determination of Mycobacterium leprae viability by use of real-time PCR.JClin Microbiol. 2009;47(7):2124-2130.

25. Liu PT, et al. MicroRNA-21 targets the vitamin D-dependent antimicrobial pathway in leprosy. Nat Med. 2012;18(2):267-273.

26. Ridley DS. Pathology and bacteriology of early lesions in leprosy. Int J Lepr Other Mycobact Dis. 1971;39(2):216-224.

27. Ridley MJ. The mononuclear cell series in leprosy: an ultrastructural report. Lepr Rev. 1981;52(1):35-50.

28. Sibley LD, Franzblau SG, Krahenbuhl JL. Intracellular fate of Mycobacterium leprae in normal and activated mouse macrophages. Infect Immun. 1987;55(3):680-685.

29. Hagge DA, Ray NA, Krahenbuhl JL, Adams LB. An in vitro model for the lepromatous leprosy granuloma: fate of Mycobacterium leprae from target macrophages after interaction with normal and activated effector macrophages. J Immunol. 2004;172(12):7771-7779.

30. Fabri M, et al. Vitamin D is required for
IFN-gamma-mediated antimicrobial activity of human macrophages. Sci Transl Med. 2011;3(104):104ra102.

31. Poli C, et al. IL-26 confers proinflammatory properties to extracellular DNA. JImmunol. 2017;198(9):3650-3661.

32. Schnaith A, Kashkar H, Leggio SA, Addicks K, Krönke M, Krut O. Staphylococcus aureus subvert autophagy for induction of caspaseindependent host cell death. J Biol Chem. 2007;282(4):2695-2706.

33. Tranchemontagne ZR, Camire RB, O'Donnell VJ, Baugh J, Burkholder KM. Staphylococcus aureus Strain USA300 perturbs acquisition of lysosomal enzymes and requires phagosomal acidification for survival inside macrophages. Infect Immun. 2016;84(1):241-253.

34. Jubrail J, et al. Inability to sustain intraphagolysosomal killing of Staphylococcus aureus predisposes to bacterial persistence in macrophages. Cell Microbiol. 2016;18(1):80-96.

35. Stenger S, Rosat JP, Bloom BR, Krensky AM, Modlin RL. Granulysin: a lethal weapon of cytolytic T cells. Immunol Today. 1999;20(9):390-394.

36. Ochoa MT, et al. T-cell release of granulysin contributes to host defense in leprosy. Nat Med. 2001;7(2):174-179.

37. Walch $\mathrm{M}$, et al. Perforin enhances the granulysin-induced lysis of Listeria innocua in human dendritic cells. BMC Immunol. 2007;8:14.

38. Montoya D, et al. Divergence of macrophage phagocytic and antimicrobial programs in leprosy. Cell Host Microbe. 2009;6(4):343-353.

39. Job CK, Jayakumar J, Aschhoff M. "Large numbers" of Mycobacterium leprae are discharged from the intact skin of lepromatous patients; a preliminary report. Int J Lepr Other Mycobact Dis. 1999;67(2):164-167.

40. Van Voorhis WC, et al. The cutaneous infiltrates of leprosy: cellular characteristics and the predominant T-cell phenotypes. N Engl JMed. 1982;307(26):1593-1597.

41. Sieling PA, et al. Immunosuppressive roles for IL-10 and IL-4 in human infection. In vitro modulation of T cell responses in leprosy. J Immunol. 1993;150(12):5501-5510.

42. Krutzik SR, et al. Activation and regulation of Toll-like receptors 2 and 1 in human leprosy. Nat Med. 2003;9(5):525-532.

43. Edfeldt K, et al. T-cell cytokines differentially control human monocyte antimicrobial responses by regulating vitamin D metabolism. Proc Natl Acad Sci U S A. 2010;107(52):22593-22598.

44. Schenk M, et al. NOD2 triggers an interleukin-32-dependent human dendritic cell program in leprosy. Nat Med. 2012;18(4):555-563.

45. Berry MP, et al. An interferon-inducible neutrophil-driven blood transcriptional 
signature in human tuberculosis. Nature. 2010;466(7309):973-977.

46. Maertzdorf J, et al. Common patterns and disease-related signatures in tuberculosis and sarcoidosis. Proc Natl Acad Sci U S A. 2012;109(20):7853-7858.

47. Frehel C, Rastogi N. Mycobacterium leprae surface components intervene in the early phagosome-lysosome fusion inhibition event. Infect Immun. 1987;55(12):2916-2921.

48. Silva BJ, et al. Autophagy is an innate mechanism associated with leprosy polarization. PLoS Pathog. 2017;13(1):e1006103.

49. Watson RO, Manzanillo PS, Cox JS. Extracellular M. tuberculosis DNA targets bacteria for autophagy by activating the host DNA-sensing pathway. Cell. 2012;150(4):803-815.

50. Abdallah M, Emam H, Attia E, Hussein J, Mohamed N. Estimation of serum level of interleukin-17 and interleukin-4 in leprosy, towards more understanding of leprosy immunopathogenesis. Indian J Dermatol Venereol Leprol. 2013;79(6):772-776.

51. Saini C, Ramesh V, Nath I. CD4+ Th17 cells discriminate clinical types and constitute a third subset of non Th1, Non Th2 T cells in human leprosy. PLoS Negl Trop Dis. 2013;7(7):e2338.

52. Chaitanya VS, Jadhav RS, Lavania M, Singh $\mathrm{M}$, Valluri V, Sengupta U. Interleukin-17F single-nucleotide polymorphism $(7488 \mathrm{~T}>\mathrm{C})$ and its association with susceptibility to leprosy. Int $J$ Immunogenet. 2014;41(2):131-137.

53. Gerosa F, et al. Differential regulation of interleukin 12 and interleukin 23 production in human dendritic cells. JExp Med. 2008;205(6):1447-1461.

54. Khader SA, et al. IL-23 compensates for the absence of IL-12p70 and is essential for the IL-17 response during tuberculosis but is dispensable for protection and antigen-specific IFN-gamma responses if IL-12p70 is available. JImmunol. 2005;175(2):788-795.

55. Freches D, Korf H, Denis O, Havaux X, Huygen $\mathrm{K}$, Romano M. Mice genetically inactivated in interleukin-17A receptor are defective in longterm control of Mycobacterium tuberculosis infection. Immunology. 2013;140 (2):220-231.

56. Umemura $\mathrm{M}$, et al. IL-17-mediated regulation of innate and acquired immune response against pulmonary Mycobacterium bovis bacille Calmette-Guerin infection. J Immunol. 2007;178(6):3786-3796.

57. Khader SA, et al. IL-23 and IL-17 in the establishment of protective pulmonary CD4+ T cell responses after vaccination and during Mycobacterium tuberculosis challenge. Nat Immunol. 2007;8(4):369-377.

58. Ma CS, et al. Deficiency of Th17 cells in hyper IgE syndrome due to mutations in STAT3. JExp Med. 2008;205(7):1551-1557.

59. de Beaucoudrey L, et al. Mutations in STAT3 and IL12RB1 impair the development of human IL-17-producing T cells. J Exp Med. 2008;205(7):1543-1550.

60. You W, et al. IL-26 promotes the proliferation and survival of human gastric cancer cells by regulating the balance of STAT1 and STAT3 activation. PLoS ONE. 2013;8(5):e63588.

61. Tameris MD, et al. Safety and efficacy of MVA85A, a new tuberculosis vaccine, in infants previously vaccinated with BCG: a randomised, placebo-controlled phase $2 \mathrm{~b}$ trial. Lancet. 2013;381(9871):1021-1028.

62. Bleharski JR, et al. Use of genetic profiling in leprosy to discriminate clinical forms of the disease. Science. 2003;301(5639):1527-1530.

63. Lahiri R, Randhawa B, Krahenbuhl J. Application of a viability-staining method for Mycobacterium leprae derived from the athymic $(\mathrm{nu} / \mathrm{nu})$ mouse foot pad. JMed Microbiol. 2005;54(Pt 3):235-242.

64. Nilsson IM, Hartford O, Foster T, Tarkowski A. Alpha-toxin and gamma-toxin jointly promote Staphylococcus aureus virulence in murine septic arthritis. Infect Immun. 1999;67(3):1045-1049.

65. Schindler D, et al. Dendritic cells are central coordinators of the host immune response to Staphylococcus aureus bloodstream infection. Am J Pathol. 2012;181(4):1327-1337.

66. Ridley DS, Jopling WH. Classification of leprosy according to immunity. A five-group system. Int J Lepr Other Mycobact Dis. 1966;34(3):255-273. 